Portland State University

PDXScholar

12-12-1994

\title{
On Modeling of a Mobile Multipath Fading Channel
}

Heng Xiao

Portland State University

Follow this and additional works at: https://pdxscholar.library.pdx.edu/open_access_etds

Part of the Electrical and Computer Engineering Commons Let us know how access to this document benefits you.

\section{Recommended Citation}

Xiao, Heng, "On Modeling of a Mobile Multipath Fading Channel" (1994). Dissertations and Theses. Paper 5054.

https://doi.org/10.15760/etd.6930

This Thesis is brought to you for free and open access. It has been accepted for inclusion in Dissertations and Theses by an authorized administrator of PDXScholar. Please contact us if we can make this document more accessible: pdxscholar@pdx.edu. 


\section{THESIS APPROVAL}

The abstract and thesis of Heng Xiao for the Master of Science in Electrical and

Computer Engineering were presented December 12, 1994, and accepted by the thesis committee and the department.

COMMITTEE APPROVALS:

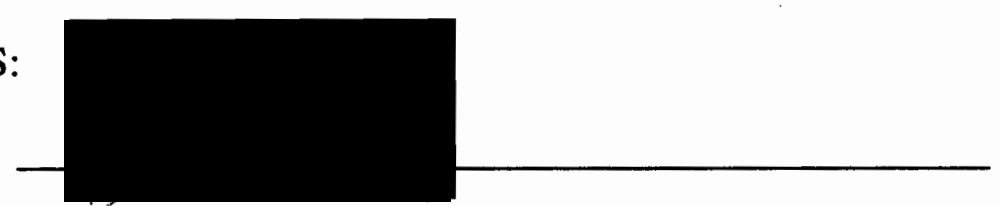

Fu Li, Chair

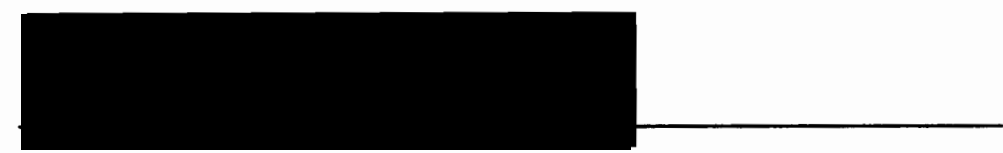

Richard D. Morris

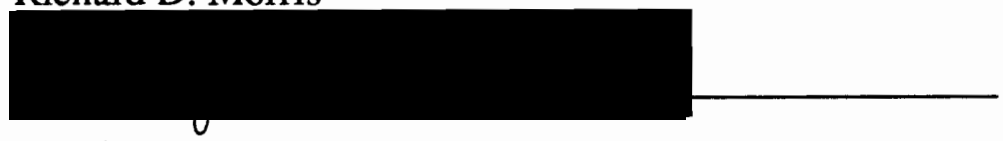

Bradford R. Crain

\section{DEPARTMENT APPROVAL:}

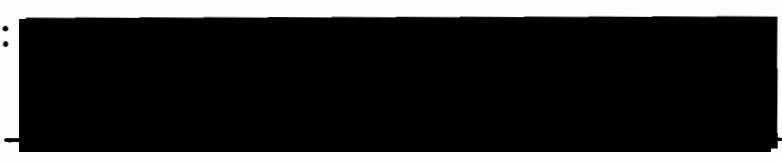

Rolf Schaumann, Chair

Department of Electrical Engineering

$* * * * * * * * * * * * * * * * * * * * * * * * * * * * * * * * * * * * * * * * * * * * * * * *$ 


\section{ABSTRACT}

An abstract of the thesis of Heng Xiao for the Master of Science in Electrical and Computer Engineering presented December 12, 1994, and accepted by the thesis committee and the department.

Title: On Modeling of a Mobile Multipath Fading Channel

Multipath fading is one of the major practical concerns in wireless communications. A multipath transmission takes place when a transmitted signal arrives at a receiver by two or more paths of different delay. Such multiple paths may be due to atmospheric reflection or refraction, or reflections from buildings or other objects.

A multipath fading channel is usually modeled as a time-variant tapped delay system. RAKE receiver can be used to detect the signal from the multipath fading channel. However, the number of delay taps, the time delay of each path, and the tap weight for each delay path need to be determined.

The objective of this thesis is to explore the possibility of using the advance signal processing algorithms to estimate the number of delays, the time delay of each path, and the tap weight for each delay path in multipath channels and to investigate the performance of the RAKE receiver based on chip rate channel estimates in a realistic mobile environment.

Simulations show that the new approach outperforms the existing approaches 
ON MODELING OF A MOBILE MULTIPATH FADING CHANNEL

by

HENG XIAO

A thesis submitted in partial fulfillment of the requirements for the degree of

\author{
MASTER OF SCIENCE \\ in \\ ELECTRICAL AND COMPUTER ENGINEERING
}

Portland State University

1995 


\section{ACKNOWLEDGEMENTS}

I wish to express my deepest thanks and gratitude to my advisor, Dr. Fu Li, for his invaluable guidance, encouragement and unrelenting patience during the course of this research.

My sincere appreciation goes to the other members of the committee, Drs. Richard D. Morris and Bradford R. Crain for their helpful comments and understanding. I wish to thank Dr. Jin Yang at SHARP Microelectronics Technology, Inc. for providing the insightful guidance and giving me valuable advice and encouragement in the course of this research. I want to thank my classmate Yibing Guo for his co-research work. Thanks are also due to other members of the faculty and staff at Portland State University who have contributed greatly to my graduate education.

Finally, my parents, my wife, and my daughter, deserve my special thanks for their continued support, encouragement and sacrifice throughout, and I will forever be indebted to them for all that they have done. 
TABLE OF CONTENTS

PAGE

ACKNOWLEDGMENTS ....................................................................... iii

LIST OF TABLES …............................................................................ vii

LIST OF FIGURES _............................................................................... viii

\section{CHAPTER}

I INTRODUCTION ..................................................................... 1

I.1 Thesis Outline .......................................................................... 2

I.2 Notation ......................................................................... 3

II MOBILE COMMUNICATION ………………........................... 5

II.1 Transmission Media _.............................................................. 6

II.2 Digital Multiplexing ............................................................. 6

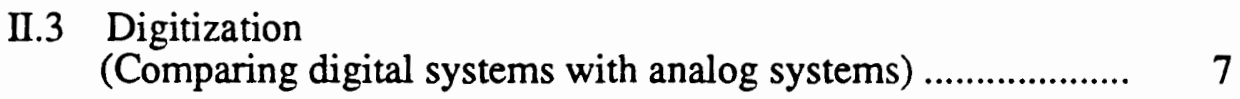

II.4 Digital Radio System Configuration ………………................. $\quad 9$

II.5 Data Communications ......................................................... 12

II.6 Modulation Methods …......................................................... 13

II.7 Error Control (Detection and Correction) ................................ 17

II.8 Types of Mobile Systems ......................................................... 19

II.9 Compare With Access Techniques

(for Mobile Communications FDMA, TDMA and CDMA) .... 21

III MULTIPATH FADING ………................................................ 24

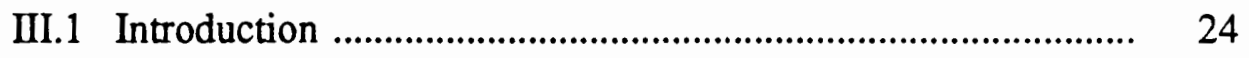




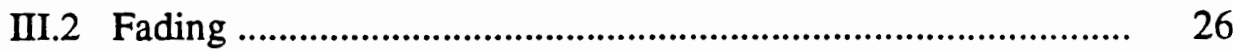

III.3 Long-Term Fading, $m(t)$ or $m(x)$........................................ 26

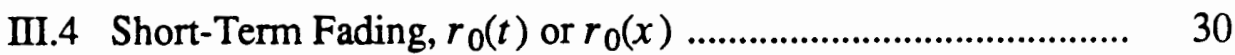

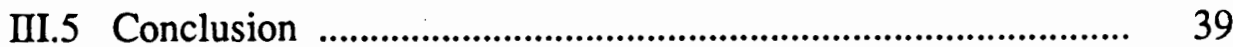

IV RAKE RECEIVER _................................................................ 40

IV.1 Introduction ........................................................................ 40

IV.2 General Principles of RAKE Design ...................................... 41

IV.3 RAKE Receiver ................................................................ 45

IV.4 Conclusion ........................................................................ 51

V DETECTION : AIC AND MDL APPROACH …………................. 53

V.1 Introduction ..................................................................... 53

V.2 IS-95 Standard …............................................................. 55

V.3 Pilot Channel .................................................................. 56

V.4 Bit-Error-Rate (BER) ........................................................ 58

V.5 Multipath Fading Channel ................................................... 59

V.6 DETECTION : AIC and MDL Approach ............................... 61

V.7 Performance Comparison ........................................................ 63

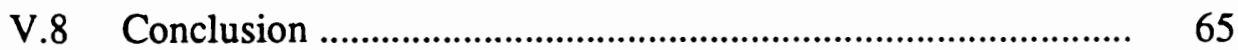

VI ESTIMATION : MUSIC AND MIN-NORM APPROACH .............. 66

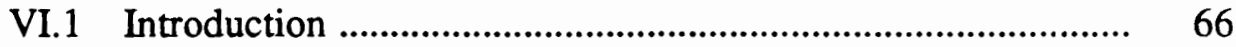

VI.2 Multipath Fading Channel ...................................................... 67

VI.3 ESTIMATION : MUSIC and MIN-NORM Approach ........... 69

VI.4 Performance Comparison …..................................................... 70

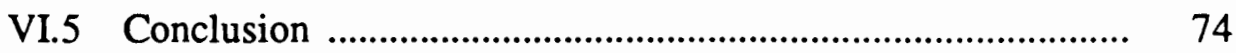

VII TAP WEIGHTS ESTIMATION .................................................. 75

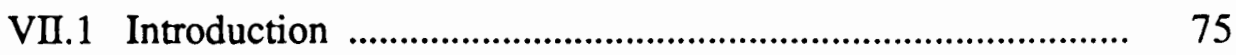


VII.2 Diversity Receiver ...................................................... 77

VII.3 New Approach ......................................................... 78

VII.4 Performance Comparison ................................................. 82

VII.5 Conclusion .................................................................. 85

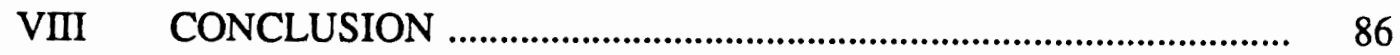

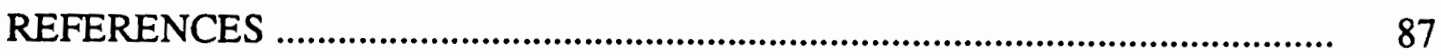




\section{LIST OF TABLES}

TABLE

PAGE

2.7.1 Convolution Encoder Bit Sequences ................................................ 19

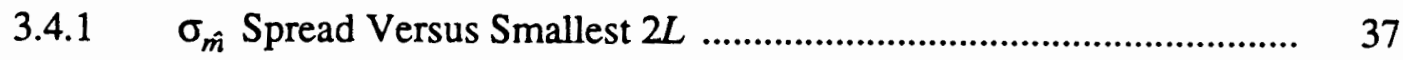

5.2.1 Rayleigh distribution ................................................................... 55 


\section{LIST OF FIGURES}

FIGURE

PAGE

2.4.1 Basic Microwave Link Incorporating A Repeater.

2.4.2 Comparison Between Analog (A) and Digital (B)

Microwave Radio Transmitters.

2.4.3 Comparison Between Analog (A) and Digital (B)

Microwave Radio Transmitters.

2.4.4 A Regenerative Repeater.

11

2.6.1 Two-Phase Shift Keying. .......................................................... 14

2.6.2 Pulse Response For An Ideal and Practical Low-Pass Filter. ........... 16

2.6.3 Filter Characteristics. $f_{n}=f_{s} / 2$;

$f_{s}=1 / T_{s}=$ Symbol Transmission Rate.

2.7.1 One-Half Rate, $K=3$ Convolution Encoder.

2.8.1 Types of Cellular Radio Systems. 20

3.1.1 Description of a Mobile Radio Environment. 25

3.3.1 The Local Mean and Terrain Contuor.

3.3.2 (A) Inverse Bending, Substandard Conditions;

(B) Positive Bending, Superstandard Conditions.

3.4.1 Mechanics of Multipath Fading.

3.4.2 The Mobile Receiver is Moving ( No Scatterers ).

3.4.3 A Reception from $N$ Reflected Waves.

3.4.4 Rayleigh Distribution of pdf. ................................................. 35

4.2.1 Tapped Delay Line Model of Multipath-Fading Channel. ................ 45 
4.3.1 Optimum Demodulator for Wideband Signal. ................................ 46

4.3.2 Optimum Demodulator for Delay Received Signal Configuration. .. 49

5.1.1 Tap Weight Estimation. .......................................................... 54

IS-95 Standard. ................................................................ 55

5.5.1 Tapped Delay Model for Multipath Fading. ................................. 59

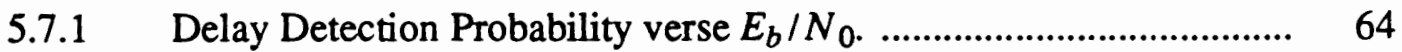

6.2.1 Tapped Delay Model for Multipath Fading. .................................. 67

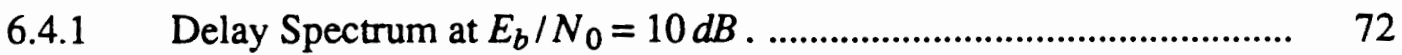

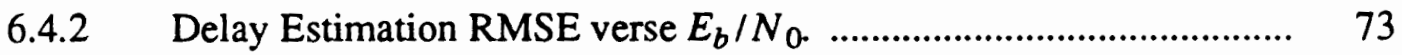

7.1.1 Tap Weight Estimation. ......................................................... 76

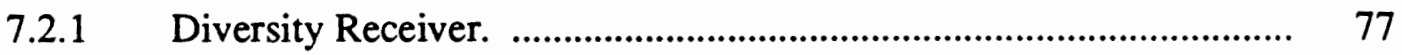

7.3.1 Simulation Block Diagram. ...................................................... 78

7.3.2 Estimation Tap Weights using Pilot Channel. ............................... 80

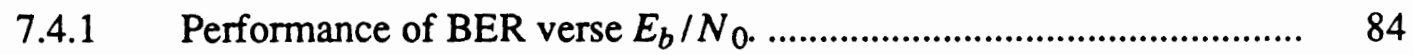




\section{CHAPTER I}

\section{INTRODUCTION}

Multipath fading is one of the major practical concerns in wireless communications. A multipath transmission takes place when a transmitted signal arrives at receiver by two or more paths of different delays. Such multiple paths may be due to atmospheric reflection or refraction, or reflections from buildings or other objects. In mobile environment, the source of multipaths normally attributes to the surroundings to the mobile unit. The different paths may consist of several discrete paths, or might consist of a continuum of paths [2]. In a building-up area, there may not even be a line-of-sight path (direct path) from the vehicle-borne antenna to the base-station transmitter. Propagation is therefore mainly by way of scattering from the surface of the buildings and by diffraction over and/or around them.

In a multipath environment, the signals arrive from different directions each with different attenuation and different time delay. They combine vectorially at receiver to give a resultant signal which fluctuates in its level. Without correction of such fading, multiple paths will generally cause severe degradation the quality of the mobile communications.

Multipath fading channel is usually modeled as a time-variant tapped delay system $[12,13]$. Based on this model, a diversity receiver can be used to detect the signal from the multipath fading channel. However, the number of delay taps, the time delay of each paths, and the tap weight for each delay path need to be determined. 
The objective of this thesis is to explore the possibility of using the advance signal processing algorithms to estimate the number of delay, the time delay of each paths, and the tap weight for each delay path in multipath channels and to investigate the performance of the diversity receiver based on chip rate channel estimates in a realistic mobile environment.

\section{I.1 THESIS OUTLINE}

This thesis is organized as follows:

Chapter I : General introduction.

Chapter II : We review mobile communication first, then describe the basic concepts of mobile communication such as transmission media, digital multiplexing, digitization. Two types of mobile communications systems, analog mobile communications systems and digital mobile communications systems, are discussed. comparison of FDMA, TDMA and CDMA is discussed.

Chapter III : As mobile radio signal, both long-term fading signal and short-term fading signal are introduced to understand the statistical characteristics of fading signals. A particular class fading signal functions and mathematical algorithms are presented. The characterization of Rayleigh statistical model is discussed.

Chapter IV : As a optimum receiver, $R A K E$ receiver is introduced and is used to detect the signal from the multipath fading channel based on the tapped delay line model. The error rate performance of the $R A K E$ receiver is discussed.

Chapter V : We propose to use array processing to determine the number of delay paths (AIC and MDL methods). 
Chapter VI : We propose to use spectrum analysis to estimate the time delay of each path (MUSIC and Min-Norm methods).

Chapter VII : We propose the new tap weight estimation method for the RAKE receiver to estimate tap weight of each delay path based on chip rate channel information (using LSE method) in a realistic mobile environment. An wide-band spread spectrum technique is used to provides effective diversity gain to improve system performance. Simulations show that the new approach outperforms the existing approaches.

Chapter VIII : General conclusion.

\section{I.2 NOTATION}

$E$ denotes expectation.

The expected value of $X$, designated by $E(X)$, is

$$
M_{1}=E(X)=\int_{-\infty}^{\infty} x p(x) d x=m_{x}
$$

By extension, the expected value of $X^{n}$ is

$$
E\left(X^{n}\right)=\int_{-\infty}^{\infty} x^{n} p(x) d x
$$

The variance, $\sigma_{x}^{2}$, of the random variable $X$ is defined as

$$
\sigma_{x}^{2}=E\left(X-m_{x}\right)^{2}=\int_{-\infty}^{\infty}\left(x-m_{x}\right)^{2} p(x) d x
$$

Biased Time Average: When a random process $x(t)$ is recorded in a time scale, the average can be obtained as 


$$
\hat{x}(t)=\frac{1}{T} \int_{0}^{T} x(t) d t
$$

Two complex-valued functions $f_{1}(t)$ and $f_{2}(t)$ are defined to be orthogonal over the interval $\left(t_{1}, t_{2}\right)$ if

$$
\int_{t_{1}}^{t_{2}} f_{1}(t) f_{2}^{*}(t) d t=\int_{t_{1}}^{t_{2}} f_{1}^{*}(t) f_{2}(t) d t=0
$$

The left pseudo-inverse $\dagger$ of $V$ is defined as

$$
V^{\dagger}=\left(V^{H} V\right)^{-1} V^{H}
$$

The covariance matrix of $\mathrm{R}$ is defined as

$$
\operatorname{Cov}=\frac{1}{N} R R^{H}
$$




\section{CHAPTER II}

\section{MOBILE COMMUNICATION}

Mobile systems are perhaps the most exciting telecommunication development since the invention of the telephone.

It has been estimated that telecommunications market in 1990 was in the region of US $\$ 500$ billion and projections indicate this could move to US\$1 trillion by the year 2000. One tends to consider the subject of telecommunications in relation to the developed, or industrialized, world without realizing that this comprises the minority of the world's population.

Cellular mobile telephone systems are difficult to classify. They could be considered to be part of the local loop since they extend out to the subscriber handset. Because of the distances traveled between a fixed subscriber and the mobile subscriber (or mobile-to-mobile subscriber), they could be called long-haul circuits. The technology incorporates some of the most advanced radio transmission techniques. In addition, the call processing requires high-level digital switching techniques to locate the mobile subscriber and setup and to maintain calls while the mobile subscriber is in transit.

There are three generations of cellular radio systems: analog FM, narrow-band TDMA, and wide-band CDMA. The analog has been around for a number of years, narrow-band TDMA is currently being put into service, and wide-band CDMA is possibly on the horizon.

The objective of the following sections is to provide some insight into the present 
and future technological trends and also to give technical details of many aspects of present-day, high-usage, digital telecommunications transmission equipment.

\section{II.1 TRANSMISSION MEDIA}

The telecommunications objective is to produce high-quality voice, video, and data communication between any pair of desired locations, whether the distance between locations is 1 or $10,000 \mathrm{~km}$. The distance between two locations determines the type of transmission equipment used for setting up the interconnection. Communication within a building, described as a local area network (LAN), is done using metallic wires or optical fibers. Any routing of information within the building is done by a switch on the premises known as a private branch exchange (PBX).

When the distance is extended to a neighboring building or to span a distance within a village, town, or city, the local telephone network is usually used.

\section{II.2 DIGITAL MULTIPLEXING}

The process of digital multiplexing, which is a means of combining voice, video, and data channels into one composite signal ready for transmission over the microwave radio or optical fiber link. This composite signal is usually referred to as the baseband (or $\mathrm{BB}$ ). Techniques known as multiplexing were subsequently devised for passing multiple simultaneous telephone calls (referred to as traffic) down one pair of copper wires.

Telecommunications involves more than voice telephone interconnectivity. Data information transmission is becoming an increasingly important telecommunications requirement. 


\section{II.3 DIGITIZATION}

( Comparing digital systems with analog systems)

The major quality improvement obtained in digital transmission systems is due to the receiver signal recovery technique (i.e., regeneration). In analog transmission systems, each repeater retransmits the received signal and also retransmits the noise. The noise accumulates at each repeater, so after a certain transmission length the signal-tonoise ratio $(\mathrm{S} / \mathrm{N})$ will be so poor that communication is not possible. In digital transmission systems, each repeater regenerate the original received signal and retransmits the signal free of noise. Theoretically, therefore, digital transmission has no transmission length limit. However, in reality, there is a phenomenon known as jitter.

Jitter accumulates because of its introduction by several electronic circuits within a digital transmission systems. Excessive jitter causes unacceptable bit errors to occur and therefore limits the maximum link length capability of the digital system. In analog systems the $\mathrm{S} / \mathrm{N}$ determines the quality of the link or channel. In digital system, it is the bit error ratio (BER) that determines the quality of the link or channel. For example, a BER of $1 \times e^{-6}$ means that 1 bit per million transmitted bits has been received incorrectly. To summarize, the advantages of the digital systems over analog are:

1) All subscriber services such as telephony, telegraphy, high-speed data, TV, facsimile, etc., can be sent via the same transmission medium. Consequently, the concept of the integrated services digital network (ISDN) can be realized.

2) Utilization of the higher unused radio frequency (RF) bands. RF bands in the region of $10 \mathrm{GHz}$ or above are unsuitable for analog systems because of high attenuation by rain, fog, etc. Whereas the $\mathrm{S} / \mathrm{N}$ in analog systems decreases 
linearly with fading of the RF carrier, the BER in digital systems is unaffected by fading until the received RF level abruptly approaches the threshold value.

3) High immunity against noise makes digital transmission almost independent of link length.

4) Use of integrated circuits makes digital systems economical and alignment free.

5) Easy maintenance, based on go/no-go type of measurement.

6) Synergistic integration of digital transmission systems such as optical fiber, digital satellite, and digital microwave radio systems with digital exchanges.

As networks become more digitized, the combination of the time-division multiplexing (TDM), time-division switching, digital radio, and optical fiber systems is considerably more economical and technically flexible than the corresponding analog networks.

The digital baseband signal transmitted by digital microwave radios or optical fiber line terminal equipment is formed by TDM instead of analog frequency division multiplexing (FDM) as it was previously. This digital multiplexer equipment is considerably simpler and cheaper than its analog counterpart. The TDM technique involves periodically sampling numerous channels and interleaving them on a time basis. The resulting transmitted bit stream of $1 \mathrm{~s}$ and $0 \mathrm{~s}$ is unraveled at the receiving end so that the appropriate bits are allocated to the correct channels to reconstruct the original signal. 


\section{II.4 DIGITAL RADIO SYSTEM CONFIGURATION}

Fig. 2.4.1 shows a simplified microwave link incorporating just one regenerative repeater and two end terminal stations. The terminal stations house switching exchanges which connect the customers to the long distance paths. In this illustration, a large number of customers (e.g., around 2000) are multiplexed together ready for transmission over the microwave link. The signal is converted to the microwave frequency (around 6 $\mathrm{GHz}$ ) and transmitted over a path of typically 30 to $60 \mathrm{~km}$ from station $A$ to the receiving antenna at repeater station. station B receives the microwave signal, processes it, and unravels the individual channels ready for distribution to the appropriate customers at this end of the link

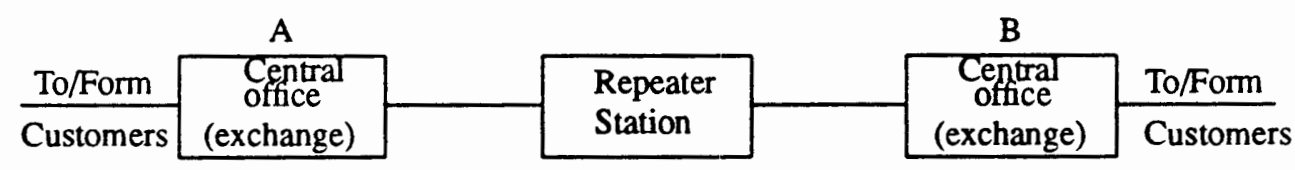

Fig. 2.4.1 Basic microwave link incorporating a repeater.

Fig. 2.4.2 is a simplified block diagram showing the major differences between analog microwave radio (AMR) and digital microwave radio (DMR) transmitters. At the intermediate frequency (IF), the two systems are very similar. The IF-to-RF conversion shown here is done by the heterodyne technique. the modulated signal is mixed with an $\mathrm{RF}$ local oscillator to form the RF signal which is then amplified and filtered ready for transmitting from the antenna.

The baseband is the combined, multiple voice, data and/or video channels which are to be transmitted over a telecommunications transmission system. 

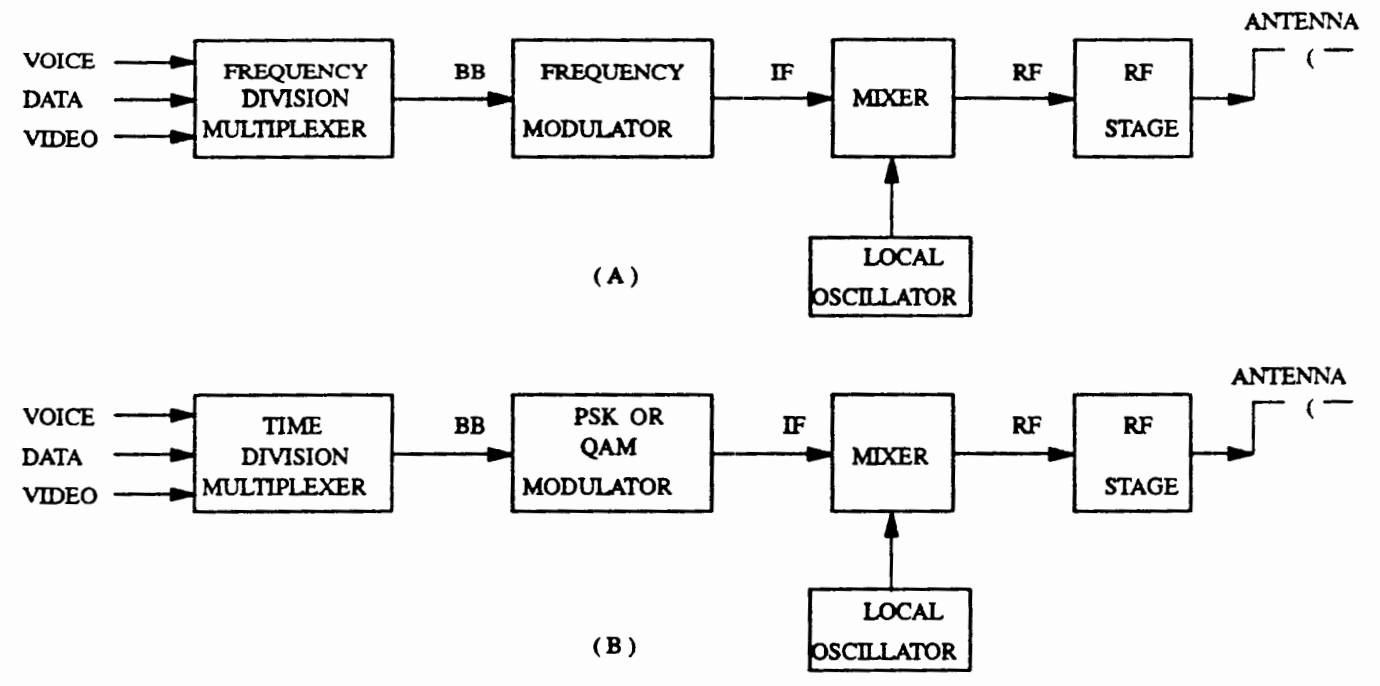

Fig. 2.4.2 Comparison between analog (A) and

digital (B) microwave radio transmitters.

As indicated in the simplified diagram of Fig. 2.4.3, the receivers differ mainly in the demodulation technique and demultiplexing of the baseband down to the voice, data, or video channels. For the AMR receivers $(\mathrm{Rx})$, the incoming $\mathrm{RF}$ signal is downconverted, frequency demodulated, and then frequency division demultiplexed to separate the individual voice, data, or video channels.

For the DMR receiver, the incoming RF signal is similarly down-converted to an prior to demodulation. Coherent demodulation is preferred. However, for coherent demodulation, exact transmitted carrier frequency and phase of the modulated signal must be obtained at the receiver. The demodulated signal is subsequently restored to its original transmitted bit stream of pulses by the regenerator. Finally, the time-division demultiplexer separates the individual voice, data, or video channels for distribution to their appropriate locations. 
ANTENNA

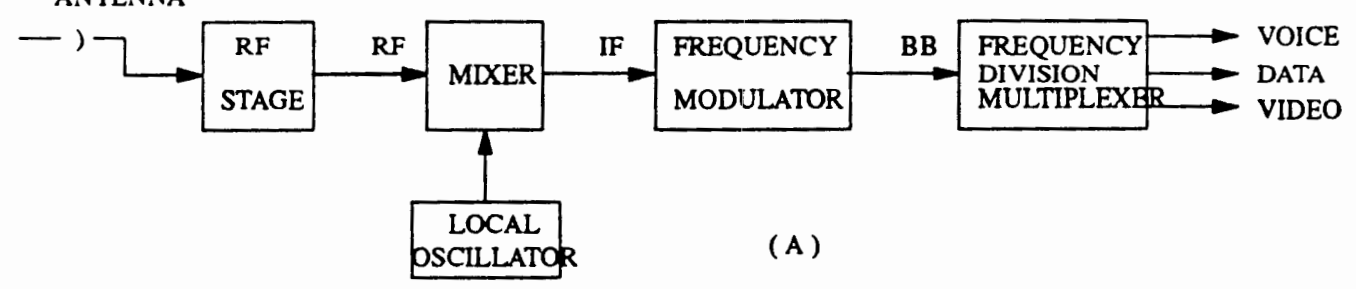

ANTENNA

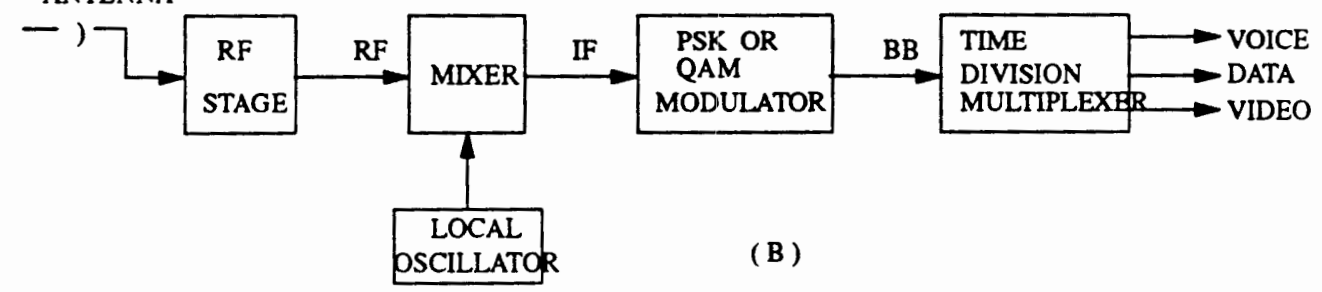

Fig. 2.4.3 Comparison between analog $(\mathrm{A})$ and digital (B) microwave radio receivers

Long distance DMR link systems use regenerative repeaters. Regenerative is a term used when the signal goes through a complete demodulation-regeneration-modulation process (Fig. 2.4.4). Regenerators can interface with digital multiplex equipment for data insert and drop applications. In regenerative repeaters the noise and distortion is largely removed in the regeneration process and so there is no noise accumulation, only jitter accumulation.

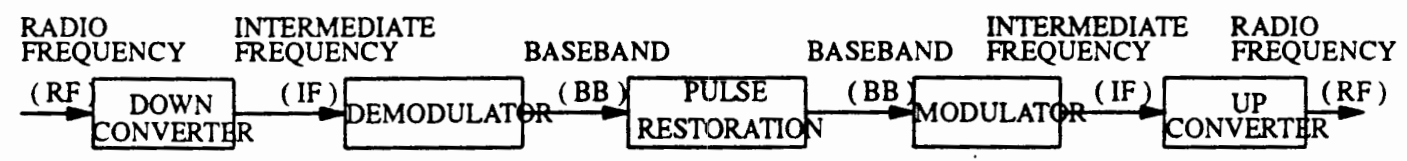

Fig. 2.4.4 A regenerative repeater 


\section{II.5 DATA COMMUNICATIONS}

The transmission of digital information is considered to be a bit stream, which is a sequence of millions of $1 \mathrm{~s}$ and 0 s that represent a combination of many voice and data channels or a video channel. The information to be communicated starts as analog information which is digitized for transmission then reconverted to analog signals at the destination. There are inevitable analog-to-digital(A/D) and digital-to-analog(D/A) conversions in this type of network. While telephone traffic was, and still is, the primary aim of the telecommunications network, a growing presence of data traffic has developed.

There is a fundamental problem which arises when trying to merge data transmission into the telephone network designed for analog telephone traffic- bandwidth. The telephone network was initially designed to have $300-$ to $3400-\mathrm{Hz}$ bandwidth for a voice channel. This is not compatible with megabit- per-second data rates (bit rates) which are ideally required by computers. Fortunately, the rates achievable at present are fast enough to allow a reasonable amount of information transfer, but the required data rates are increasing rapidly. The obvious solution that springs to mind is to use a wide bandwidth transmission medium such as optical fiber cable for data transmission. The bandwidth capability of the optical fiber would allow a variety of services to be provided including video-phone, video for TV, stereo music channels, etc., Such as a broad-band integrated services digital network (B - ISDN). an interim ISDN has been proposed which extends a $64-\mathrm{kb} / \mathrm{s}$ path out to the customers' premises. This is a considerable improvement over the early data bit rates of 9.6 or $14.4 \mathrm{~kb} / \mathrm{s}$ using $A / D$ and $D / A$ converters, called modems, over an analog telephone network 3.1-kHz bandwidth circuit.

Instantaneous processing and transmission are required so that there is no irritating delay as is sometimes experienced on satellite links. There is an important difference here when considering data which does not have this real-time constraint. Instead 
of using the conventional speech circuit switching, data can be split up into packets and transmitted when convenient time slots become available in a time division multiple access(TDMA) type of medium. This packet switching has become a significant force in data communications today [1] .

\section{II.6 MODULATION METHODS}

The methods of modulation used in analog communication systems can be broadly categorized as amplitude modulation (AM) and frequency modulation (FM). Digital communication systems follow a different approach. The signal to be transmitted in a digital system is a stream of $1 \mathrm{~s}$ and $0 \mathrm{~s}$. There are only two amplitude levels, $O N$ or $O F F$. At first sight this appears to be a much simpler problem to solve than the transmission of an analog signal whose amplitude is varying in a very complex manner. Unfortunately, a pulse is composed of a fundamental tone plus an infinite number of harmonics. Theoretically, that requires an infinite bandwidth for the transmission of a single pulse. Any communication system is limited in available bandwidth, and it is this constraint that causes a considerable complexity in the design of digital modulators and demodulators (modems).

There are several modulation techniques, which can be broadly categorized as follows:

1) Pulse amplitude modulation (PAM)

2) Frequency shift keying (FSK)

3) Phase shift keying (PSK)

4) a mixture of phase and amplitude modulation, called quadrature amplitude 
modulation (QAM)

PSK modulation is widely used in DMR technology today. There are several levels of PSK. The simplest is two-phase PSK (BPSK) as shown in Fig. 2.6.1A. In this type of modulation, the incoming bit stream is given a phase reversal of $180^{\circ}$ every time a 1 changes to a 0 or vice versa. Fig. 2.6.1B shows the waveform changing between $0^{0}$ and $180^{\circ}$. The next level of PSK is four-phase FSK, otherwise known as quadrature or quaternary (QPSK or 4-PSK).

In general, the M level of PSK is M-PSK.

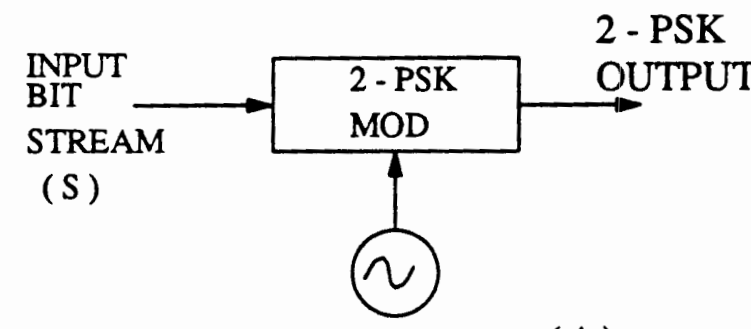

(A)
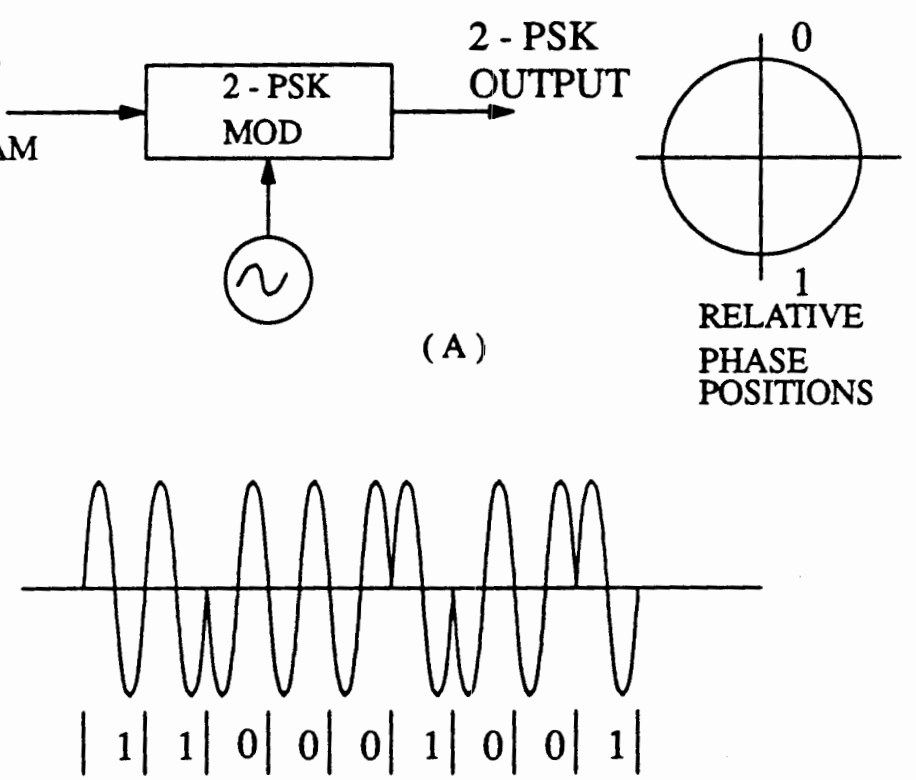

CARRIER PHASE CHANGES

(B)

Fig. 2.6.1 Two-phase shift keying

In the modulation process, We have to briefly indicate the effects of filters used in the modulation process on the bandwidth efficiency of a digital signal. Since the bandwidth required to perfectly transmit pulses is infinite, if the available bandwidth is 
comparatively narrow, there will be a significant effect on the shape of pulses emerging from a bandwidth-limiting device such as a filters

\section{FILTER}

The use of low-pass or bandpass filters in the modulation or up-conversion processes of a communication systems is unavoidable. Passing the pulses through a lowpass or bandpass filters will eliminate some components of the pulses, resulting in output pulses having very rounded corners instead of sharp, right-angle corners. Eventually, if the cut-off frequency of a low-pass filter reaches a low enough value, the pulses become so rounded that they do not reach their amplitude. The Nyquist Theorem states that if pulses are transmitted at a rate of $f_{s} b / s$, they will attain the full amplitude value if passed through a low-pass filter having a bandwidth $f_{s} / 2 \mathrm{~Hz}$. This is the minimum filtering requirement for pulse transmission without performance degradation (i.e., no intersymbol interference). Fig. 2.6.2 illustrates this ideal Nyquist filter, which allows pulses to reach their maximum amplitudes. Unfortunately, this type of ideal Nyquist filter does not exist. If it did, it would require an infinite number of filter sections, which would therefore have an infinite cost. The filter characieristics for transmission of impulses (approximately the same as for very narrow pulses) are shown in Fig. 2.6.3. The value of $\alpha=0$ is the ideal filter case. A more practical value of $\alpha=0.3$ requires a bandwidth of 30 percent in excess of the Nyquist bandwidth. This means that instead of transmission at an ideal bandwidth efficiency of $2 \mathrm{~b} / \mathrm{s} / \mathrm{Hz}$, the value is $2 / 1.3=1.54$ b/s/Hz. Fig. 2.6.2 also shows the output response [1] . 

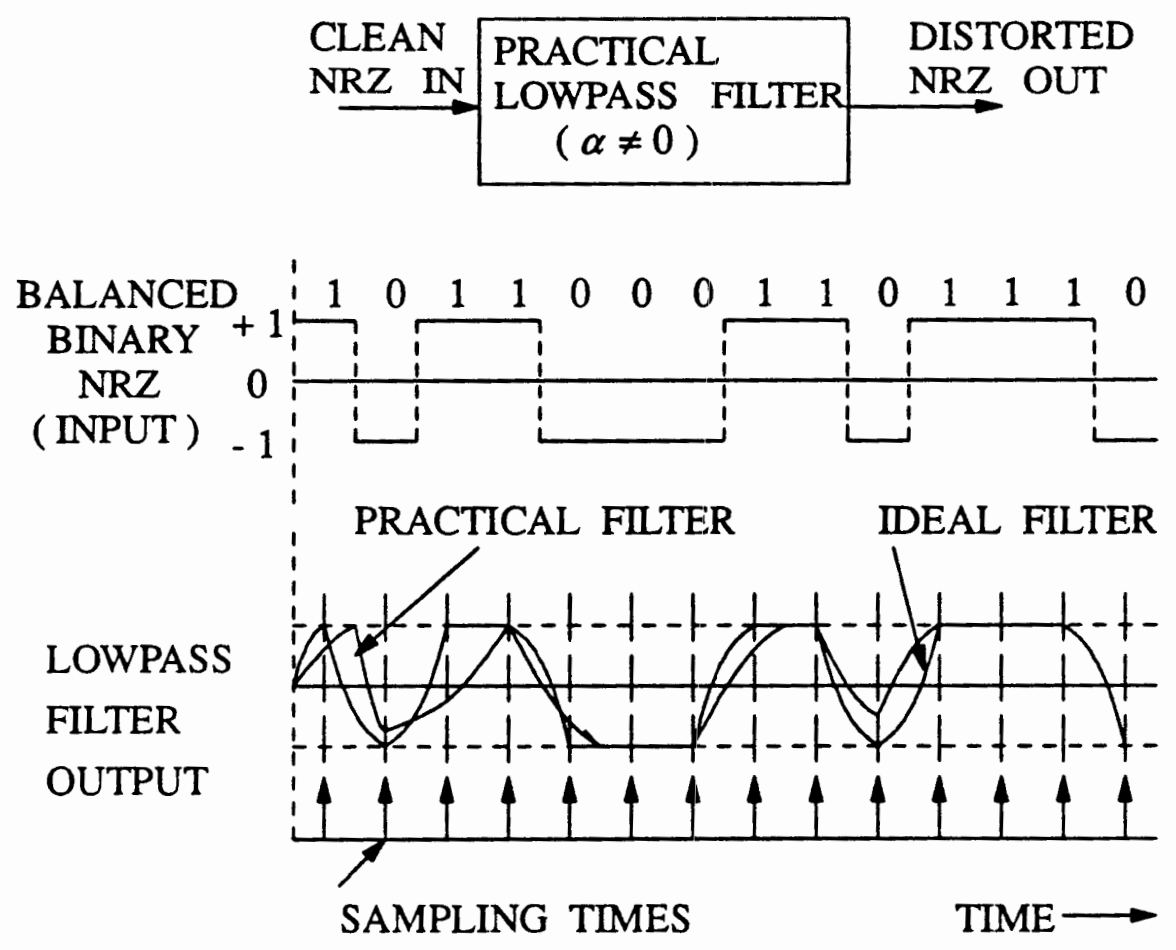

Fig. 2.6.2 Pulse response for an ideal and practical low-pass filter.

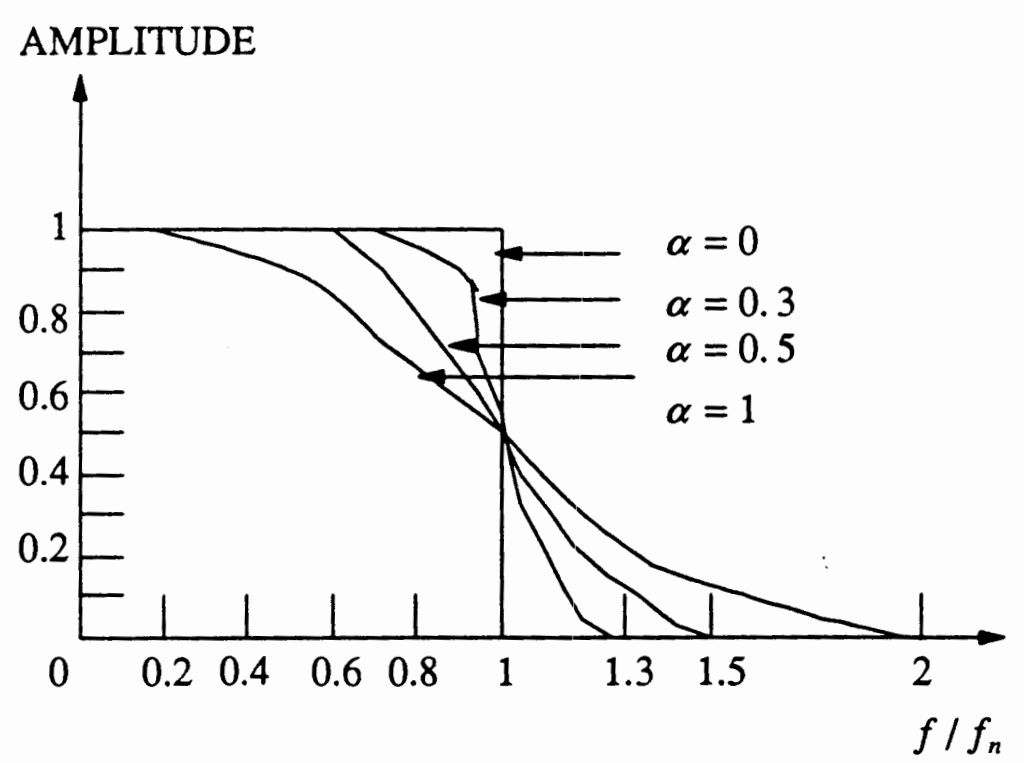

Fig. 2.6.3 Filter characteristics. $f_{n}=f_{s} / 2 ; f_{s}=1 / T_{s}=$ symbol transmission rate. 


\section{II.7 ERROR CONTROL (DETECTION and CORRECTION)}

The BER is perhaps the most important quality factor observe when evaluating a digital transmission system. First, it is instructive to make a statement about the noise. Error calculations are often based on the type of random noise described as additive white gaussian noise (AWGN).

The probability of receiving errors for a specific modulation scheme depends on the $\mathrm{S} / \mathrm{N}$ at the receiver and the transmission bit rate. In practical system, there is usually no opportunity to change those two factors, so one must resort to error control coding, also called channel coding, or broadly speaking, FEC. Recent developments in incorporating error control into the modulation process have produced some exciting results. This technique is called trellis-coded modulation (TCM). TCM achieve improved noise immunity (low residual BER) with a minimal reduction in bandwidth efficiency. Error correction codes can be broadly categorized into

1) block codes

2) convolution codes

3) a combination of both block and convolution, called concatenated codes

In here, we mainly consider convolution codes. The convolution code was the first type of coding to receive widespread acceptance, and it was initially used in satellite transmission applications.

For the convolution code-word, the encoder depends on the block of message bits $(k)$ and the previous $(n-1)$ blocks of message bits. This rather complex process is performed by shift registers and adders. 
Fig. 2.7.1 illustrates a convolution encoder using only three shift registers.

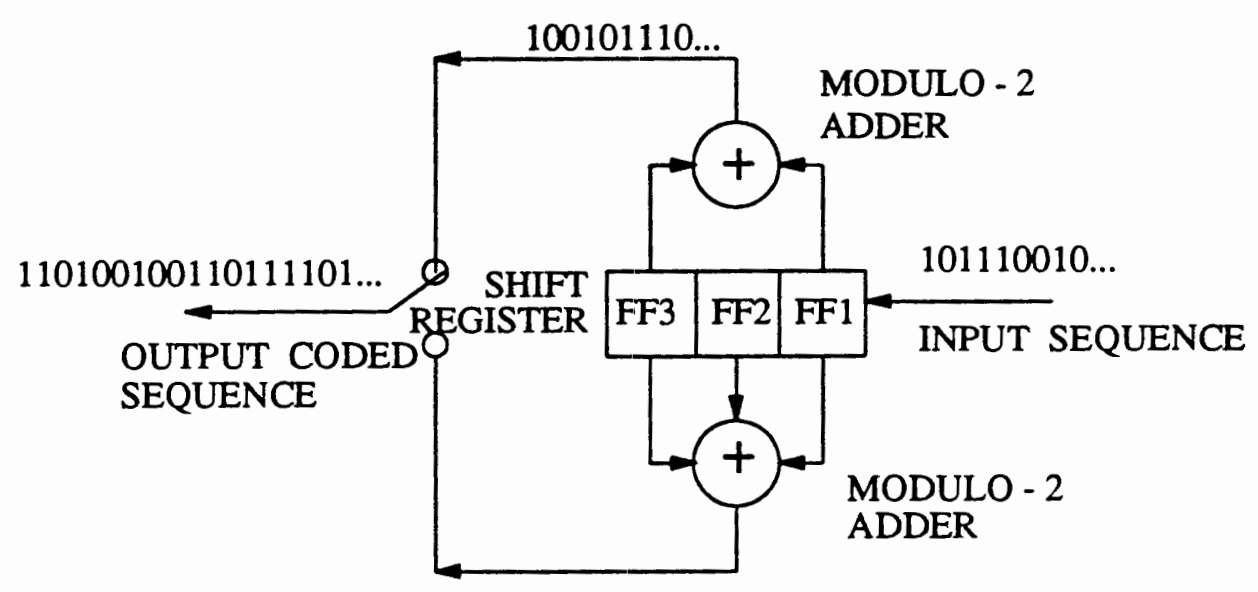

Fig. 2.7.1 One-half rate, $K=3$ convolution encoder

The number of shift registers forming the encoder is called the constraint length $K$, so Fig. 2.7.1 has $K=3$. The input data is gradually shifted through the registers, and the outputs are fed into the two modulo- 2 adders as indicated. A modulo- 2 adder, by definition, produces a 1 output for an odd number of 1 inputs. A simple truth table check for two inputs to a modulo-2 adder confirms it to be the familiar EXCLUSIVE OR function. The switch moves back and forth to take alternate bits from each modulo-2 adder output to produce the encoded output. In this case there are 2 coded bits for every input data bit. This is called a $1 / 2$ - rate encoder. The code rate in general in $1 / n$, where $n$ is the number of coded output bits per input data bit. For example, consider an input data sequence of 101110010 . The outputs of the modulo- 2 adders are shown in Table 2.7.1 (considering the shift registers initially set at 000 and the sequence was followed by zeros). The switch then takes alternate bits to form the encoded output:

$$
110100100110111101 \ldots
$$


TABLE 2.7.1 CONVOLUTION ENCODER BIT SEQUENCES

\begin{tabular}{|c|c|c|}
\hline $\begin{array}{l}\text { OUTPUT FROM } \\
\text { IP ADDER }\end{array}$ & SHIFT REG. VALUES & $\begin{array}{l}\text { OUTPUT FROM 2 } \\
\text { IP ADDER }\end{array}$ \\
\hline 1 & 001 & 1 \\
1 & 010 & 0 \\
0 & 101 & 0 \\
0 & 011 & 1 \\
1 & 111 & 0 \\
0 & 110 & 1 \\
1 & 100 & 1 \\
1 & 001 & 1 \\
1 & 010 & 0 \\
\hline
\end{tabular}

\section{II.8 TYPES OF MOBILE SYSTEMS}

There are three generations of cellular radio systems: analog FM, narrow-band TDMA, and wide-band CDMA.

Analog FM The first-generation cellular systems in operation were analog FM radio systems which allocated a single carrier for each call. Each carrier was frequency modulated by the caller. The carriers were typically spaced at $25 \mathrm{kHz}$ intervals (i.e., carrier bandwidth). The allocated bandwidth was relatively narrow, and only a few channels (typically 12) were available.

Digital FDMA The FDMA systems resemble analog FM, with the exception that the carrier is modulated by a digitally encoded speech signal. The bandwidth of each carrier is similar to the analog FM systems (i.e., $25 \mathrm{kHz}$ ).

Digital narrow-band TDMA The TDMA systems operate with several customers sharing one carrier. Each user is allocated a specific time slot for transmission and reception of short bursts or packets of information. The bandwidth of each carrier is typically $200 \mathrm{kHz}$, and the total bandwidth available is in the region of 10 to $30 \mathrm{MHz}$, 
which allows a reasonably large channel capacity in the region of 500 to 1000 channels.

Digital wide-band CDMA One form of digital wide-band operation which has good future potential is CDMA. In these systems there is a single carrier which is modulated by the speech signals of many users. Instead of allocating each user a different time slot, each is allocated a different modulation code. Mobile users in adjacent cells all use the same frequency band. Each user contributes some interfering energy to the receivers of the fellow users, the magnitude of which depends on the processing gain.

The different types of mobile system, in terms of frequency spectrum usage, are summarized in Fig. 2.8.1. They differ primarily in modulation technique and carrier spacing.

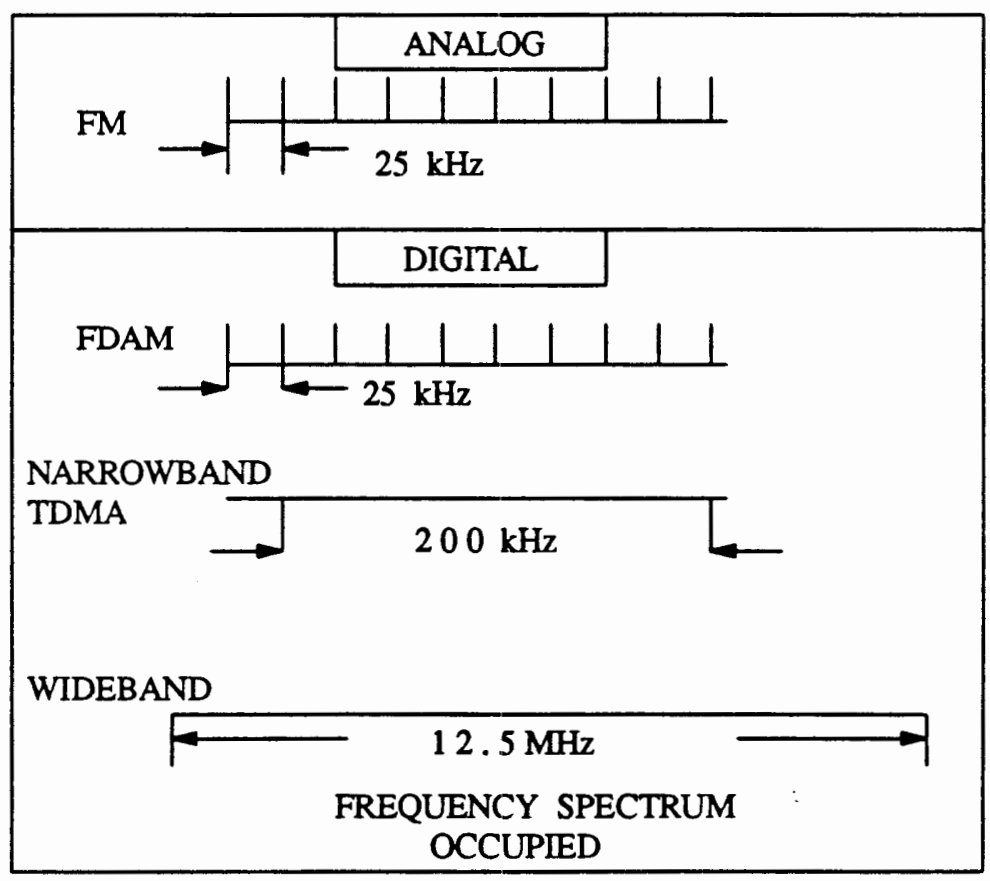

Fig. 2.8.1 Types of cellular radio systems. 


\section{II.9 COMPARE WITH ACCESS TECHNIQUES}

(for Mobile Communications: FDMA, TDMA and CDMA )

Both frequency division multiple access (FDMA) and (TDMA) are widely used for digital transmission, a very brief distinction between the two will now be clarified for the mobile communications application.

Broadly speaking, FDMA simply means splitting up an available frequency band into a specific number of channels, and the bandwidth of each channel depends on the type of information signals to be transmitted. One pair of channels is used for duplex operation. Information to be transmitted is superimposed on a carrier at the channel center frequency. The information can be a composite of several information signals, which are multiplexed prior to being superimposed on the carrier, or a single information signal can be placed on the carrier. This would be called a single channel per carrier (SCPC) system.

Initially, the analog information was superimposed on the carriers using FM. More recently, the analog signals have been converted to digital pulse streams and the PSK and QAM techniques employed.

By contrast, the TDMA scheme uses only one frequency band, and many channels are created by transmitting information for each channel in allocated time slots.

In a TDMA mobile radio system, each base station is allocated a $25-$ or $30-\mathrm{kHz}$ channel, and users share this same channel on a time-allocated basis. The maximum number of users of each channel depends on how many bits per second are required to digitize the voice of each user. the conventional voice $A / D$ conversion process requires $64 \mathrm{~kb} / \mathrm{s}$. If a digital modulation technique such as QPSK is used, each voice channel can be digitized with a bandwidth efficiency of at least $1 \mathrm{~b} / \mathrm{s} / \mathrm{Hz}$ (i.e., $8.5 \mathrm{kHz}$ of bandwidth 
is required for each digital voice signal). This means that for a $30-\mathrm{kHz}$ TDMA channel, there can be three users per channel (or twelve users per channel with degradation).

CDMA has only recently received widespread interest because of its potential benefits in mobile telecommunication systems. The objective of CDMA is to allow many users to occupy the exact same frequency band without interfering with each other. It sounds too good to be true. However, with spread spectrum knowledge, it is quite a reasonable statement. Each user is assigned a unique orthogonal code.

In a CDMA system all signals from all users will be received by each user. Each receiver is designed to listen to and recognize only one specific sequence. Having locked onto this sequence, the signal can be dispread, so the message stands out above the other signals, which appear as noise in comparison. Interference does become a limiting factor because, eventually, as more users occupy the same frequency band, the noise level rises to a point where dispersion does not provide an adequate $S / N$.

No guard time in CDMA. Guard time is required in TDMA between time slot. Guard time does occupy the time periods of certain bits. The waste bits could be used to improve quality performance in TDMA.

In CDMA guard time does not exist.

For microcell and in-building systems. CDMA is a natural waveform suitable for microcell and in-building because it is susceptible to noise and interference.

The asynchronous nature of CDMA compare to TDMA gives it the advantage that network synchronization is not required. Second, it is relatively easy to add users to a CDMA system. Third, CDMA is more tolerant to multipath fading than TDMA or FDMA.

In conclusion, CDMA has the potential to enable a more efficient use of the frequency spectrum than other techniques, and when the remaining technical problems 
have been solved, it should be particularly well suited to cellular mobile radio systems [1] . Two papers [14], [15] analyzed CDMA in depth; other interesting literature on CDMA can be found in references [16] - [24] . 


\section{CHAPTER III}

\section{MULTIPATH FADING}

\section{III.1 INTRODUCTION}

A wave propagation mechanism is closely affected by the wavelengths of the propagation frequencies. Whether suburban or urban, buildings are natural wave scatterers. The sizes of buildings are equivalent over many wavelengths of a propagation frequency, creating reflected waves at that frequency. The frequency range for a mobile radio multipath environment would be $30 \mathrm{MHz}$ and higher.

We might imagine that the mobile site will receive many reflected waves and one direct wave. The reflected waves received at the mobile site would come from different angles equally spaced throughout $360^{\circ}$, as shown in Fig. 3.1.1. Often a direct wave is presented and relatively strong as comparing with the reflected waves. The described situation is called Rician statistical model. However, a mobile communication system design cannot be based upon this optimistic situation; it is based on the case of weak or nondirect waves which normally occur at the fringe area. All the reflected waves received at the mobile unit combine to produce a multipath fading signal. This described situation is called Rayleigh statistical model. 

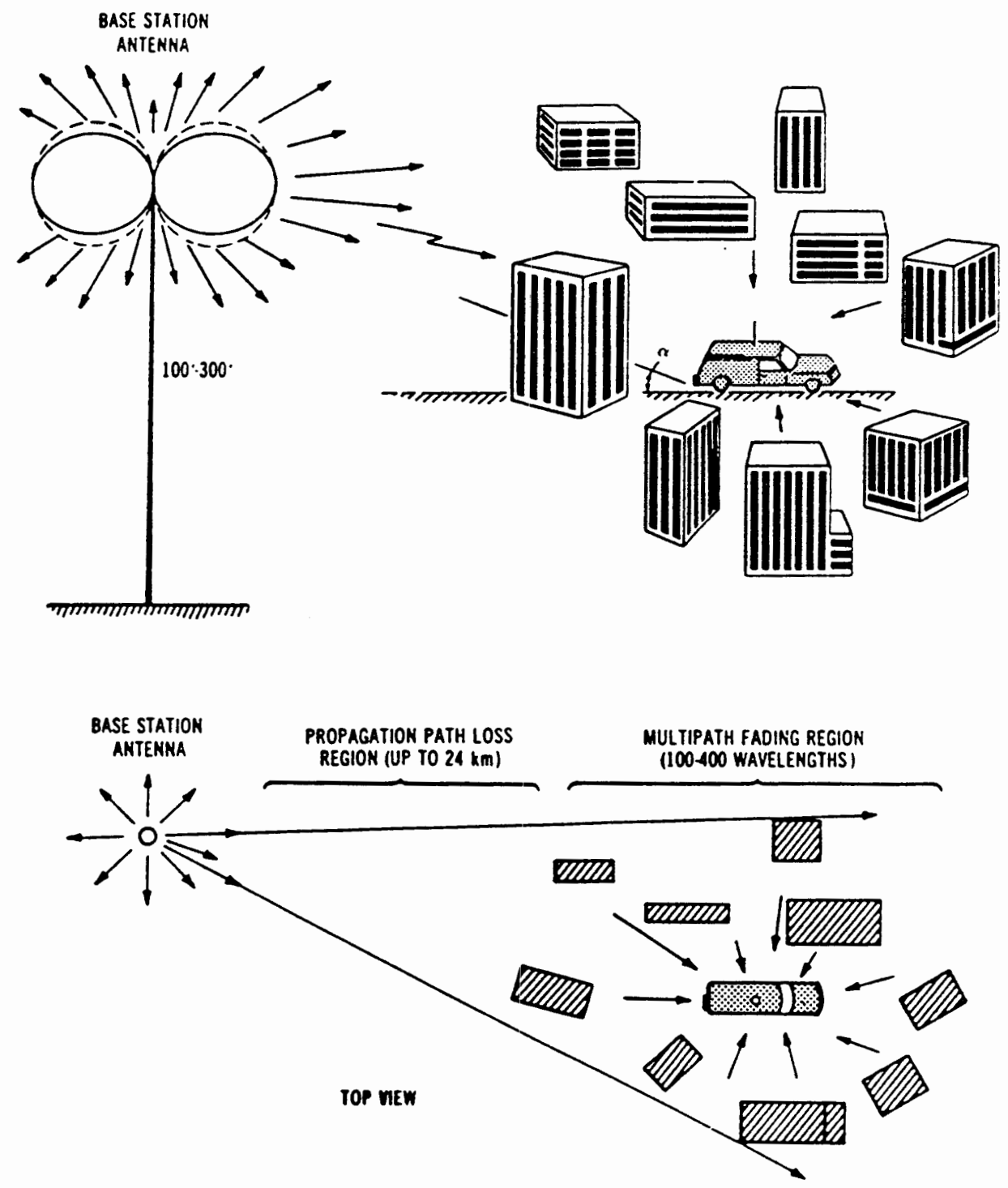

Fig. 3.1.1. Description of a mobile radio environment 


\section{III.2 FADING}

The mobile radio signal is received while the mobile unit is in motion. In this situation the fading signal of a received signal with respect to time $t$, or space $x$, is obtained. The received signal $r(t)$ can be artificially separated into two parts by cause: long-term fading $m(t)$ and short-term fading $r_{0}(t)$ as

$$
r(t)=m(t) \cdot r_{0}(t)
$$

or

$$
r(x)=m(x) \cdot r_{0}(x)
$$

Neither type of fading can be predicted accurately because each is caused by variations in atmospheric conditions. Experience has shown that some climates and terrain surfaces are more likely to cause fading than others, but in all circumstances fading can only be defined statistically. In other words, one can only say that based on probability theory the microwave system will be inoperative for a certain percentage of the year because of fading. In some regions this percentage is too large to be tolerated. Fortunately, there are techniques which can be used to improve the outage time. In here, we will mention a more detailed view of fading.

\section{III.3 LONG-TERM FADING, $m(t)$ or $m(x)$}

Long-term fading is the average or envelope of the fading signal, as the dotted curve shows in Fig. 3.3.1A. It is also called a local mean since along the long-term fading each value corresponds to the mean average of the field strength at each local point. 
The estimated local mean $\hat{m}\left(x_{1}\right)$ at point $x_{1}$ along $x$-axis can be expressed mathematically as

$$
\hat{m}(x)=\frac{1}{2 L} \int_{x_{1}-L}^{x_{1}+L} r(x) d x=\frac{1}{2 L} \int_{x_{1}-L}^{x_{1}+L} m(x) r_{0}(x) d x
$$

Assume that $m\left(x_{1}\right)$ is the true local mean, then at point $x_{1}$ in Fig. 3.3.1A.,

$$
m\left(x=x_{1}\right)=\hat{m}\left(x=x_{1}\right) \quad x_{1}-L<x<x_{1}+L
$$

When the length $L$ is properly chosen, Eq.(3.3.1) becomes

$$
\hat{m}\left(x_{1}\right)=m\left(x_{1}\right) \cdot \frac{1}{2 L} \int_{x_{1}-L}^{x_{1}+L} r_{0}(x) d x
$$

To let $\hat{m}\left(x_{1}\right)$ approach $m\left(x_{1}\right)$ in Eq.(3.3.3), the following relation holds:

$$
\frac{1}{2 L} \int_{x_{1}-L}^{x_{1}+L} r_{0}(x) d x-\cdots->1
$$

The length $L$ will be determined after fully understanding the statistical characteristics of short-term fading $r_{0}(x)$ (see III.4 CASE 5).

The long-term fading $m(x)$ is mainly caused by terrain configuration and the built environment between the base station and the mobile unit.

Terrain configurations can be classified as 
Flat terrain

Hilly terrain

Mountain area

and the human-made environment as

Rural area

Quasi-suburban

Suburban area

Urban area

The terrain configuration causes local-mean (long-term fading) attenuation and fluctuation, whereas the human-made environment only causes local-mean attenuation. The human-made environment also causes short-term fluctuation (fading) in signal reception. Under certain circumstances, the fluctuation of a long-term fading caused by the terrain configuration can form a log-normal distribution because of the statistical nature of the fluctuation shown in Fig. 3.3.1B. The probability density function (pdf) is represented as

$$
p(y)=\frac{1}{\sqrt{2 \pi} \sigma} \exp \left[-\frac{(y-m)^{2}}{2 \sigma^{2}}\right]
$$

where the parameters: log-normal variable $y$, mean $m$, and standard deviation $\sigma$ are in $\mathrm{dB}$ scale.

Experiments show that the standard deviation $\sigma$ is in the range 4 to $10 \mathrm{~dB}$. The $\sigma$ of the local-mean will vary in $\mathrm{dB}$ values dependent on the configuration of the terrain. 


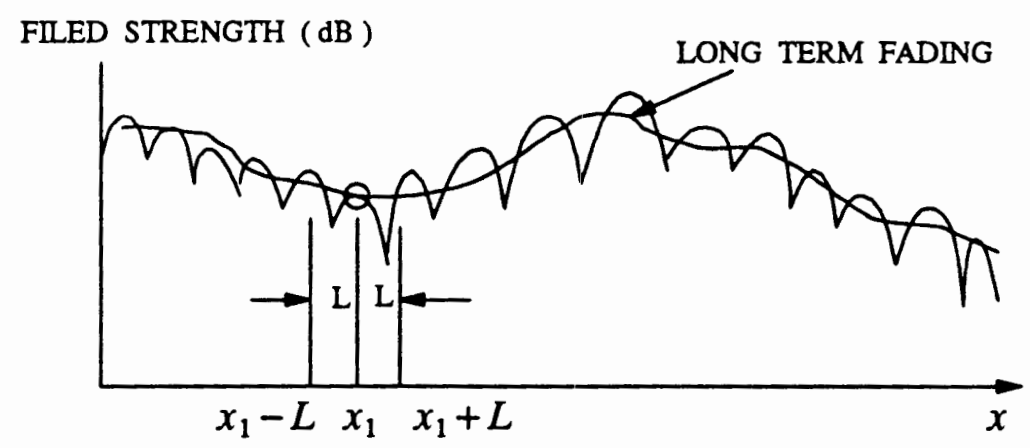

(A) The local mean, integrated from a proper $2 L$ window

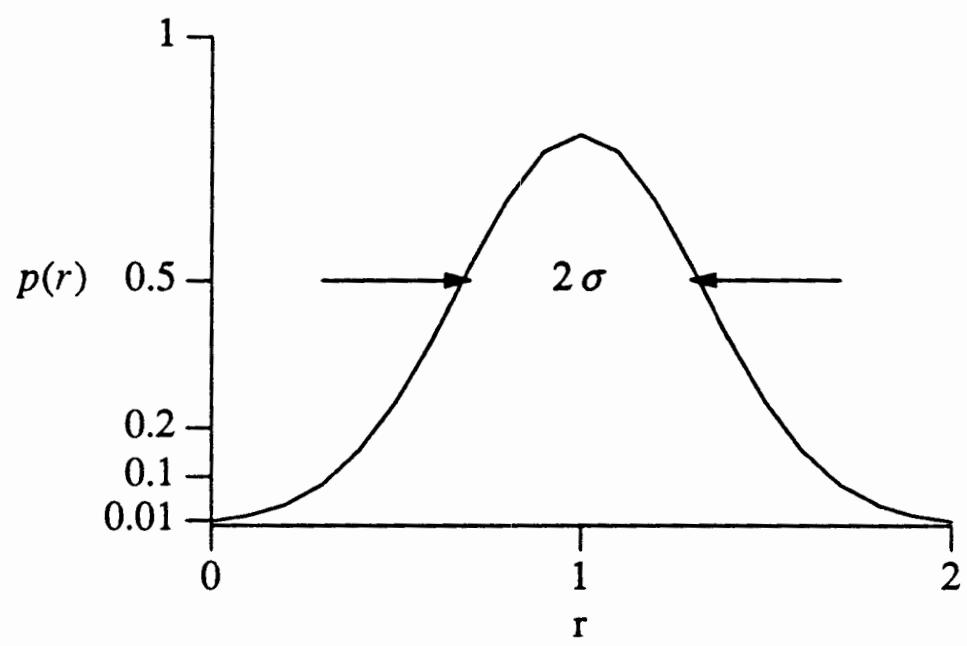

(B) The log-normal distribution

Fig. 3.3.1. The local mean and terrain contour

A more frequently occurring long-term fading is due to beam bending. the microwave beam can be influenced by a change of the refractive index (dielectric constant) of the air; $k=4 / 3$ is considered to be the standard atmospheric condition in which the microwave beam has one-fourth of the true earth curvature. When the density of the air subsequently changes such that the refractive index of the atmosphere is different from standard, the beam will be bent upward or downward, depending on the $k$-factor. 
When $k$ is less than $4 / 3$, often called a subrefractive or substandard condition, it causes upward bending, and when $k$ is greater than $4 / 3$, known as superrefractive or superstandard conditions, it causes downward bending (see Fig. 3.3.2.). The most commonly occurring type is upward bending, above the receiving antenna.

For downward bending, the fading is wide-band compared to the relatively narrow microwave frequency band (i.e., long-term fading or non-frequency-selective fading). However, if some energy is reflected from an obstacle and it interferes with the direct path energy, the fading is frequency selective.
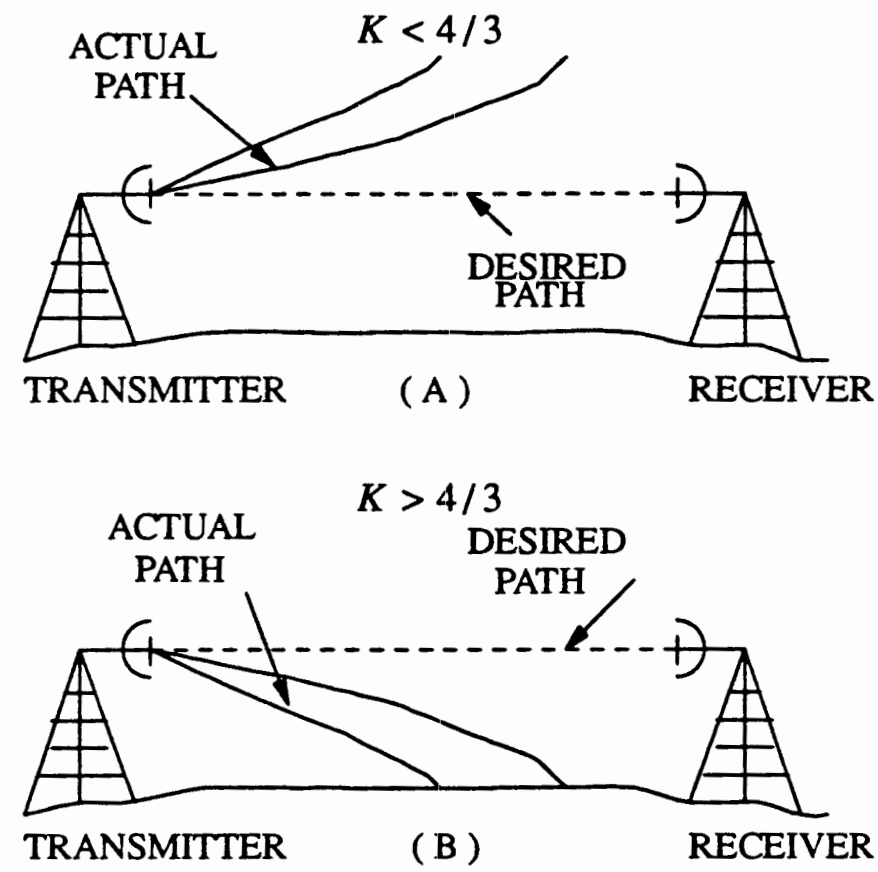

Fig. 3.3.2. (A) Inverse bending, substandard conditions;

(B) Positive bending, superstandard conditions

\section{III.4 SHORT-TERM FADING, $r_{0}(t)$ or $r_{0}(x)$}

Short-term fading is mainly caused by multipath reflections of a transmitted wave by local scatterers such as houses, buildings, and other human-built structures, or by 
natural obstacles such as forests surrounding a mobile unit. It is not caused by a natural obstruction such as a mountain or hill located between the transmitting site and the receiving site.

Short-term fading is also called multipath fading or frequency selective fading. There are some cases to illustrate the phenomenon.

\section{CASE 1. Atmospheric Selective Fading}

When the atmospheric conditions are such that layers or stratifications of different densities exist, ducting can occur. If the composition of the layers is such that the microwave beam is not trapped, but only deflected, as in Fig. 3.4.1, the microwave energy can reach the receiving antenna by paths that are different from the direct path. This multipath reception produces fading because the two waves are rarely received in phase. If they arrive in complete antiphase, for a few seconds a drop in received power, which can be $30 \mathrm{~dB}$ or more, is observed.

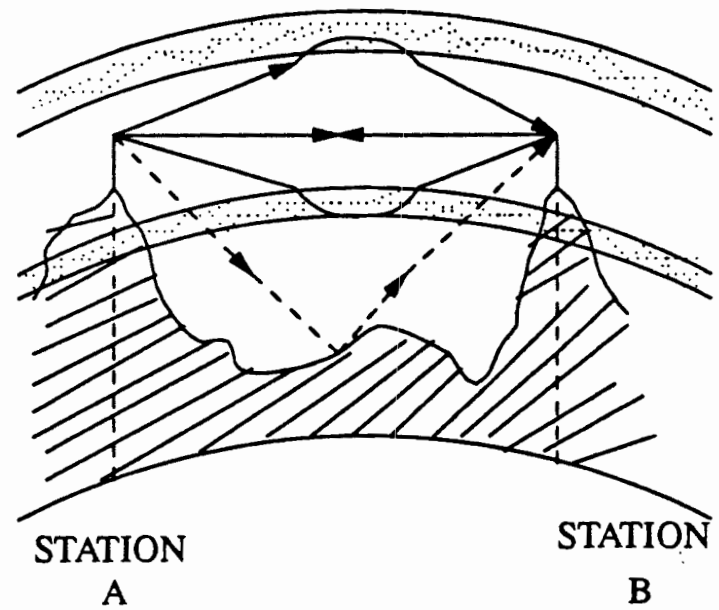

Fig. 3.4.1 Mechanics of multipath fading 
Ground reflection can cause a multipath reception which will be observed as fading if the waves are received in antiphase. When ground reflection and atmospheric multipath fading occur simultaneously, short-term fades as deep as $40 \mathrm{~dB}$ can occur. If corrective action is not taken, this will cause service disruption. Multipath fading is frequency selective because, for antiphase cancellation, the different waves must reach the receiver after traveling distances that differ by one half-wavelength. Because the size of one half-wavelength varies significantly from 1 to $12 \mathrm{GHz}$, fading conditions that exist at one frequency may not exist at another.

In general, frequency selective fading is fast fading. The average duration of a 20 $\mathrm{dB}$ fade is about $40 \mathrm{~s}$, and the average duration of a $40 \mathrm{~dB}$ fade is about $4 \mathrm{~s}$.

Experience has shown that all paths longer than $40 \mathrm{~km}$ can be subject to multipath fading for frequencies of operation above $890 \mathrm{MHz}$. As the length of a microwave path is increased, there is a rapid increase in the number of possible indirect paths by which the signal may be received. It is often necessary to reduce hop path length to $35 \mathrm{~km}$ or less to avoid serious ground reflection multipath fading.

\section{CASE 3. A General Standing Wave Condition [2]}

The mobile unit is moving with a velocity $V$, yet there is no single scatterer around the mobile unit, as shown in Fig. 3.4.2. In this case, the received signal can be represented by assuming an incoming signal arriving at an angle $\theta$ with respect to the motion of the mobile unit as

$$
S_{r}=A \exp \left[j\left(2 \pi f_{t} t-\beta x \cos \theta\right)\right]
$$

where $\beta$ is called the wave number, $\beta=2 \pi / \lambda$ and $\lambda$ is the wavelength. Expression $\left(j 2 \pi f_{t} t\right)$ is the complex exponential representing a transmitting frequency $f_{t}$ that 
propagates in time domain. $x$ is the displacement; $x=V t$, where $V$ is the speed of the mobile receiver. $A$ is a constant amplitude, and $f_{t}$ is the transmitting frequency or socall propagation frequency. Eq.(3.4.1) can be rewritten

$$
S_{r}=A \exp \left[j 2 \pi\left(f_{t}-\frac{V}{\lambda} \cos \theta\right)\right]
$$

where the Doppler frequency $f_{D}$ is

$$
f_{D}=\frac{V}{\lambda} \cos \theta
$$

The amplitude of the signal is $\left|S_{r}\right|=A$, where $A$ is a constant at the baseband. The received frequency $f_{r}$ is offset by the transmitting frequency $f_{t}$ by a Doppler frequency $f_{D}$ as

$$
f_{r}=f_{t}-\frac{V}{\lambda} \cos \theta
$$

When the mobile unit is moving away from the source $\theta=0^{0}$, the received frequency is $f_{r}=f_{t}-V / \lambda$. When the mobile unit is circling around the source $\theta=90^{\circ}$, then $f_{r}=f_{t}$. When the mobile unit is moving toward the source $\theta=180^{\circ}$, and $f_{r}=f_{t}+V / \lambda$.

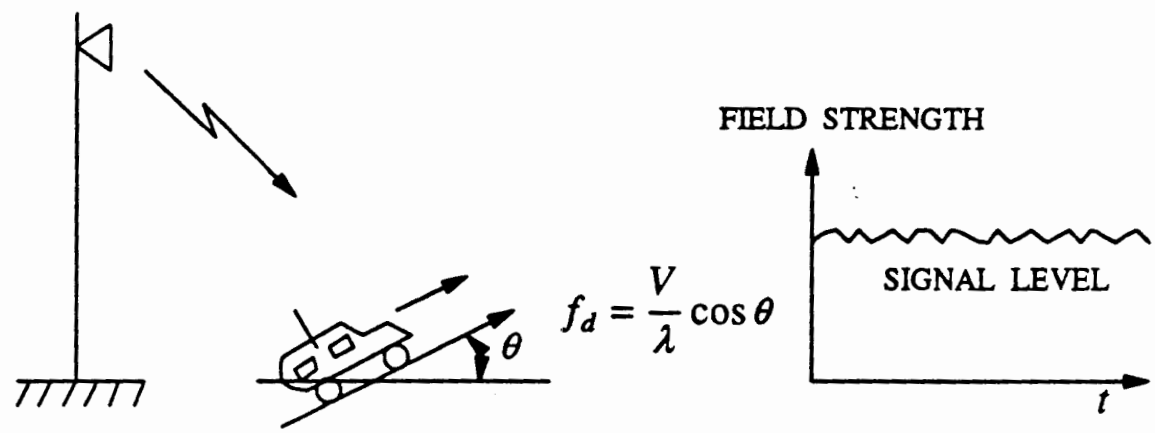

Fig. 3.4.2. The mobile receiver is moving (no scatterers) 
For $N$ reflected waves (see Fig. 3.4.3.), we have

$$
S_{r}=\sum_{i=1}^{N} A_{i} \exp \left[j 2 \pi\left(f_{t}-\frac{V}{\lambda} \cos \left(\theta_{i}\right)\right)\right]
$$

where $A_{i}$ is amplitude of $i$ 'th reflected wave, $\theta_{i}$ is the direction of $i$ 'th wave arrival.

In presenting a fading signal, $A_{i}$ is a complex random variable with zero mean and variance 1 , and $\theta_{i}$ is also a random variable from $0^{\circ}$ to $360^{\circ}$.

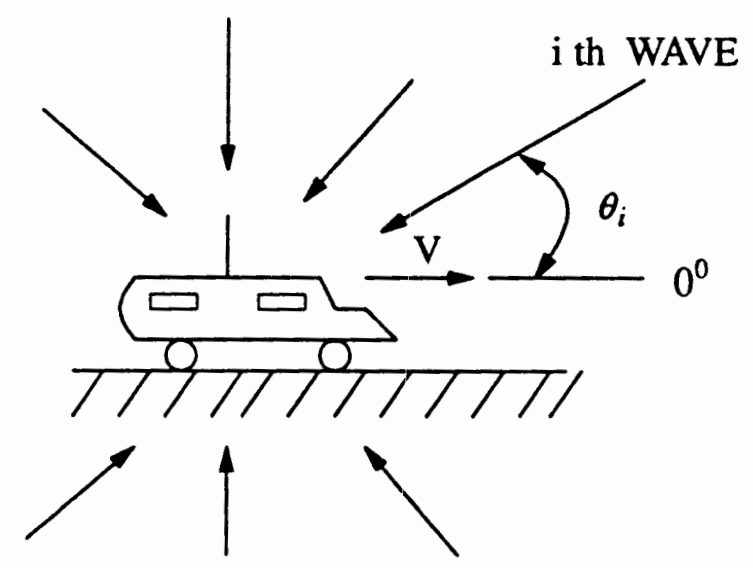

Fig. 3.4.3. A reception from $N$ reflected waves

Multipath fading can be represented by a Rayleigh distrib:tion. The probability density function:

$$
p(r)=2 \frac{r}{\text { Pave }} \exp \left(-\frac{r^{2}}{\text { Pave }}\right)
$$

where Pave $=E\left(r^{2}\right)$ is the average power of the short-term fading, or $\sqrt{\text { Pave }}$ is an rms value of $r$. The value of $r$ cannot be below zero. The Rayleigh pdf is not a symmetrical 
function (see Fig. 3.4.4.), We have, the standard deviation:

$$
\sigma_{r}=\frac{\sqrt{4-\pi}}{2} \sqrt{\text { Pave }}
$$

and the mean of $r$ is

$$
m=\frac{\sqrt{\pi}}{2} \sqrt{\text { Pave }}
$$

The probability distribution function of power is

$$
F(r)=P(x<r)=\int_{0}^{r} p(x) d x=1-\exp \left(-\frac{r^{2}}{\text { Pave }}\right)
$$

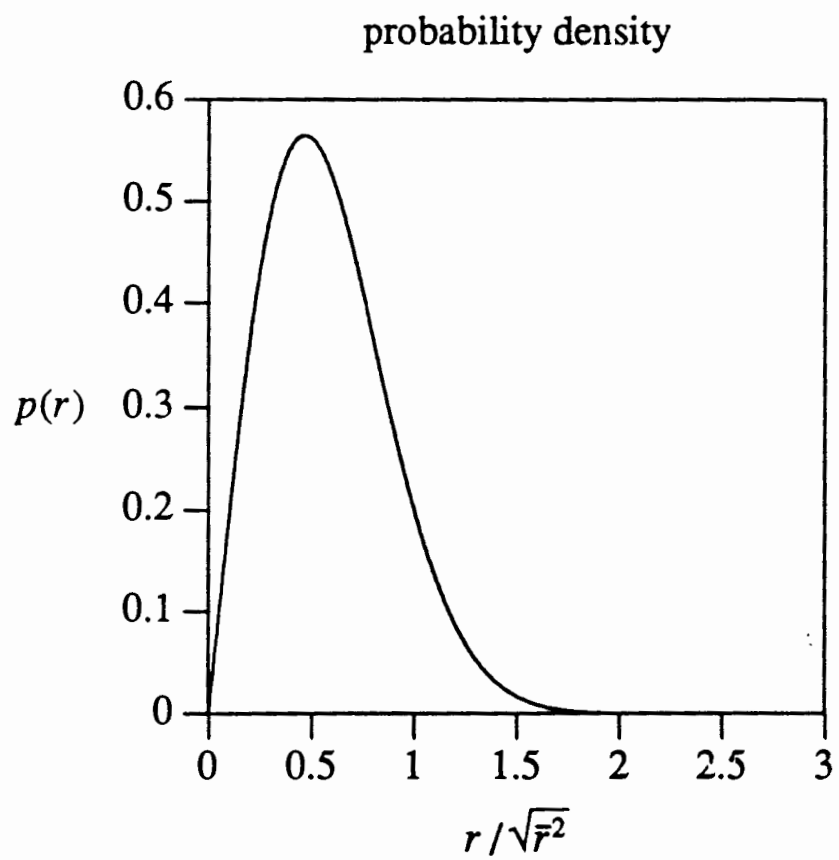

Fig. 3.4.4. Rayleigh distribution of pdf 
The fading rate is proportional to the vehicle speed and the carrier center frequency as

$$
f_{d}=\frac{\nu}{\lambda}=\frac{v}{c / f_{c}}=\frac{v}{c} f_{c}
$$

When $v=60 \mathrm{mph}$, and $f_{c}=875 \mathrm{MHz}, c$ is the speed of light $c=300,000,000$ $(\mathrm{m} / \mathrm{s})$. We have

$$
f_{d}=\frac{60 \times 1609 / 3600(\mathrm{~m} / \mathrm{s})}{\frac{300,000,000}{875,000,000}(\mathrm{~m})}=\frac{60 \times 1609 / 3600}{0.342}=78 \mathrm{~Hz}
$$

\section{CASE 4. Ergodic Process}

If a statistical average value obtained in the ensemble domain is the same as in the time domain, we call this kind of random process an ergodic process. If a communication system setup remains unchanged in an ergodic process. the data values (signal strengths of a mobile radio signal) received at each location will also remain unchanged in different time intervals. Fortunately, the mobile radio fading signal can be assumed to be an ergodic process [3].

It is easier and more effective for us to process an average in a time domain than in an ensemble domain.

\section{CASE 5. Long Term Fading $L$}

After fully understanding the statistical characteristics of short-term fading $r_{0}(x)$. we will determine the average length of long-term fading $L$.

Define a criterion :

$$
\sigma_{\hat{m}}^{2}=E\left(\hat{m}(x)^{2}\right)-E(\hat{m}(x))^{2}
$$


Assuming $r_{0}(x)$ is a Rayleigh fading shown in Eq.( 3.4.6), Inserting it in Eq.( 3.3 .3 ), we obtain

$$
E(\hat{m}(x))=\sqrt{\frac{\pi}{2}} \sqrt{\frac{\text { Pave }}{2}}
$$

Eq.(3.4.13) and Eq.(3.4.8) are the same. They show the true mean equals the mean of the sample mean. From Eq.(3.4.12), we can derive that:

$$
\sigma_{\dot{m}}^{2}=\frac{1}{4 L} \int_{0}^{2 L}\left(1-\frac{y}{2 L}\right) J_{0}^{2}(\beta y) d y
$$

The $1 \sigma_{\hat{m}}$ spread is defined as

$$
1 \sigma_{\hat{m}} \text { spread }=10 \log \frac{1+\sigma_{\hat{m}}}{1-\sigma_{\hat{m}}}(d B)
$$

Objective: to find the smallest $L$ where $\sigma_{\hat{m}}$ is smaller than $1 \mathrm{~dB}$.

The computed results of Eq.(3.4.14) and Eq.(3.4.15) are given in Table 3.4.1.

\begin{tabular}{|c|c|c|}
\hline $2 L$ & $\sigma_{\hat{m}}$ & $\sigma_{\hat{m}}$ spread $(\mathrm{dB})$ \\
\hline 5 & 0.165 & 3 \\
\hline 10 & 0.122 & 2.1 \\
\hline 20 & 0.09 & 1.56 \\
\hline 40 & 0.06 & 1 \\
\hline
\end{tabular}

Table 3.4.1. $\sigma_{\hat{m}}$ Versus $2 L$

For a spread of $1 \mathrm{~dB}, 2 L>40 \lambda$.

For a spread of $1.5 \mathrm{~dB}, 2 L>20 \lambda$. 
In practical, $L$ is between $20 \lambda$ to $40 \lambda$. where $\lambda=c / f_{c}$.

Terrain contour can change at a distance greater than $20 \lambda$ as the wavelength becomes longer.

Long-Term Fading could be removed by Auto Gain Control or other circuits. Having a length as we derived. 


\section{III.5 CONCLUSION}

Rayleigh fading is also called multipath fading in the mobile radio environment. When these multipath waves bounce back and forth due to the buildings and houses, they form many standing-wave pairs in space. Those standing-wave pairs are summed together and become an irregular wave-fading structure. When a mobile unit is standing still, its receiver only receives a signal strength at that spot, so a constant signal is observed. When the mobile unit is moving, the fading structure of the wave in the space is received. It is a multipath fading. The recorded fading beconses fast as the vehicle moves faster. 


\section{CHAPTER IV}

\section{RAKE RECEIVER}

\section{IV.1 INTRODUCTION}

The previous chapters have described mobile radio environment, mobile radio signal, multipath fading, and the statistical characterization of multipath fading channel.

In this chapter, we consider the problems of signal design, receiver structure, and receiver performance for more complex channels, namely, channels having randomly time-variant impulse responses. This characterization serves as a model for signal transmission over many radio channels such as shortwave ionospheric radio communication in the 3- to $30-\mathrm{MHz}$ frequency band (HF). The time-variant impulse responses of these channels are a consequence of the constantly changing physical characteristics of the media. For example, the ions in the ionospheric layers that reflect the signals transmitted in the HF frequency band are alway in motion. To the user of the channel the motion of the ions appears to be random. Consequently, if the same signal is transmitted at HF in two widely separated time intervals, the two received signals will not only be different, but the difference will be random rather than deterministic. The random variations that occur are treated in statistical terms.

A communication system, called $R A K E$, designed expressly to work against the combination of random multipath and additive noise disturbances. By coding the sequence of symbols to be transmitted into a wide-band signal, it becomes possible at 
the receiver to isolate those portions of the transmitted signal arriving with different delays, using correlation detection techniques [10]. A RAKE receiver can be used to detect the signal from the multipath fading channel.

\section{IV.2 GENERAL PRINCIPLES OF RAKE DESIGN}

Suppose we have decided to build a radioteletype system that will detect separately, then add up, each of the multiplicity of delayed signal arriving as a result of multipath. The transmission will then have to be wide-band, for otherwise its time waveform cannot possess sufficient detail to permit the waveform at one instant of time to be distinguished unambiguously from that at another. Naturally, the wider the bandwidth, the finer will be the time resolution.

In addition to the requirement of providing multipath resolution, the transmission must of course be capable of carrying information. In a teletype system, this is accomplished by transmitting at will distinguishable waveform, one representing the Mark baud (or signal bits), and the other Space. A commonly-used system is frequency-shift keying (FSK), where two sine waves of slightly different frequency are employed [11].

Now let us examine the effects of the channel on a transmitted signal that is represented in general as [12]

$$
s(t)=\operatorname{Re}\left[u(t) e^{j 2 \pi f_{c} t}\right]
$$

We assume that there are multiple propagation paths. Associated with each path is a propagation delay and an attenuation factor. Both the propagation delays and the attenuation factors are time-variant as a result of changes in the structure of the medium. Thus the received bandpass signal may be expressed in the form 


$$
x(t)=\sum_{n} \alpha_{n}(t) s\left[t-\tau_{n}(t)\right]
$$

where $\alpha_{n}(t)$ is the attenuation factor for the signal received on the $n$th path and $\tau_{n}(t)$ is the propagation delay for the $n$th path. Substitution for $s(t)$ from Eq.(4.2.1) into Eq.(4.2.2) yields the result

$$
x(t)=\operatorname{Re}\left(\left\{\sum_{n} \alpha_{n}(t) e^{-j 2 \pi f_{c} \tau_{n}(t)} u\left[t-\tau_{n}(t)\right]\right\} e^{j 2 \pi f_{c} t}\right)
$$

It is apparent from observation of Eq.(4.2.3) that the equivalent lowpass received signal is

$$
r(t)=\sum_{n} \alpha_{n}(t) e^{-j 2 \pi f_{c} \tau_{n}(t)} u\left[t-\tau_{n}(t)\right]
$$

Since $r(t)$ is the response of an equivalent lowpass channel to the equivalent lowpass signal $u(t)$, it follows that the equivalent lowpass channel is described by the timevariant impulse response

$$
c(\tau ; t)=\sum_{n} \alpha_{n}(t) e^{-j 2 \pi f_{c} \tau_{n}(t)} \delta\left[\tau-\tau_{n}(t)\right]
$$

Now let us consider the transmission of an unmodulated carrier at frequency $f_{c}$. Then $u(t)=1$ for all $t$ and, hence, the received signal for the case of discrete multipath, given by Eq.(4.2.4), reduces to

$$
r(t)=\sum_{n} \alpha_{n}(t) e^{-j 2 \pi f_{c} \tau_{n}(t)}=\sum_{n} \alpha_{n}(t) e^{-j \theta_{n}(t)}
$$


where $\theta_{n}(t)=2 \pi f_{c} \tau_{n}(t)$. Thus the received signal consists of the sum of a number of time-variant vectors (phasors) having amplitudes $\alpha_{n}(t)$ and phases $\theta_{n}(t)$. We note that large dynamic changes in the medium are required for $\alpha_{n}(t)$ to change sufficiently to cause a significant change in the received signal. On the other hand, $\theta_{n}(t)$ will change by $2 \pi \mathrm{rad}$ whenever $\tau_{n}$ changes by $1 / f_{c}$. But $1 / f_{c}$ is a small number and, hence, $\theta_{n}$ can change by $2 \pi$ rad with relatively small motions of the medium. $r(t)$ can be modeled as a complex-valued gaussian random process. This means that time-variant impulse response $c(\tau ; t)$ is a complex valued gaussian random in the $t$ valued gaussian random process in the $t$ variable.

The multipath propagation model for the channel embodied in the received signal $r(t)$, given in Eq.(4.2.6), results in signal fading. The fading phenomenon is primarily a result of the time variations in the phases $\left\{\theta_{n}(t)\right\}$. That is, the randomly time-variant phases $\left\{\theta_{n}(t)\right\}$ associated with the vectors $\left\{\alpha_{n} e^{-j \theta_{n}}\right\}$ at times result in the vectors adding destructively.

When the impulse response $c(\tau ; t)$ is modeled as a zero mean complex valued gaussian process, the envelope $|c(\tau ; t)|$ at any instant $t$ is Rayleigh-distributed. In this case, the channel is said to be a Rayleigh fading channel. Rayleigh-distributed envelope fading has been observed often on HF and troposcatter channel and, as a consequence, this channel model is widely accepted.

Having discussed the statistical characterization of time-variant multipath channels generally in terms of the correlation functions, let $u(t)$ be the equivalent lowpass signal transmitted over the channel and let $U(f)$ denote its frequency content. Then the equivalent lowpass received signal, exclusive of additive noise, may be expressed either in terms of the time domain variables $c(\tau ; t)$ and $u(t)$ as 


$$
r(t)=\int_{-\infty}^{\infty} c(\tau ; t) u(t-\tau) d \tau
$$

or in terms of the frequency functions $C(f ; t)$ and $U(f)$ as

$$
r(t)=\int_{-\infty}^{\infty} C(f ; t) U(f) e^{j 2 \pi f t} d f
$$

Suppose we are transmitting digital information over the channel by modulating (either in amplitude, or in phase, or both) the basic pulse $u(t)$ at a rate $1 / T$, where $T$ is the signaling interval. It is apparent from Eq.(4.2.8) that the time-variant channel characterized by the transfer function $C(f ; t)$ distorts the signal $U(f)$. If $U(f)$ has a bandwidth $W$ greater than the coherence bandwidth $(\Delta f)_{c}$ of the channel, $U(f)$ is subjected to different gains and phase shifts across the band. In such a case the channel is said to be frequency-selective. The frequency-selective depends on the multipath spread or, equivalently, on the coherence bandwidth of the channel relative to the transmitted signal bandwidth $W$.

Thus, in a frequency-selective slowly fading situation, an equivalent lowpass signal has a bandwidth $W / 2$, where $W \gg(\Delta f)_{c}$. we achieve a resolution of $1 / W$ in the multipath delay profile. Since the total multipath spread is $T_{m}$, for all practical purposes the tapped delay model for the channel can be truncated at $L=\left[T_{m} W\right]+1$ taps, where we select the signaling interval $T$ to satisfy the condition $T \gg T_{m}$ and $W$ is the signal bandwidth. The slow-fading condition implies that the channel characteristics vary sufficiently slowly that they can be measured. Then the noiseless received signal can be expressed as (see Fig. 4.2.1)

$$
r(t)=\sum_{n=1}^{L} c_{n}(t) u\left(t-\frac{n}{W}\right)+z(t)
$$


where $z(t)$ is a complex-valued zero mean white gaussian noise process, and $u(t)$ is the signal transmitted through the multipath fading channel. The time-variant tap weights $\left\{c_{n}(t)\right\}$ are zero mean complex-valued stationary gaussian random processes. The magnitudes $\left|c_{n}(t)\right| \equiv \alpha_{n}(t)$ are Rayleigh-distributed and the phases $\phi_{n}(t)$ are uniformly distributed. Since the $\left\{c_{n}(t)\right\}$ represent the tap weights corresponding to the $L$ different delays $\tau=n / W, n=1,2, \ldots, L$, the uncorrelated scattering assumption made as above implies that the $\left\{c_{n}(t)\right\}$ are mutually uncorrelated. But the $\left\{c_{n}(t)\right\}$ are gaussian random processes; hence they are statistically independent [12] .

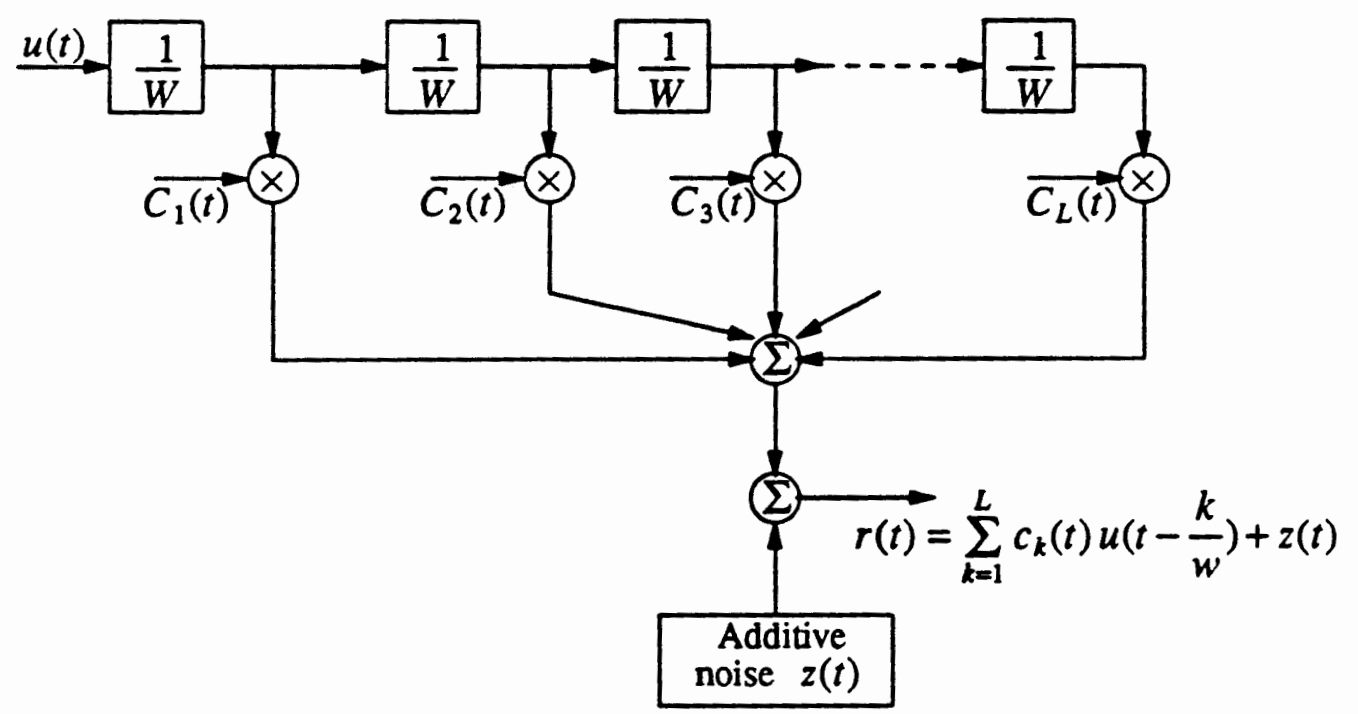

Fig. 4.2.1. Tapped delay line model of multipath-fading channel.

\section{IV.3 RAKE RECEIVER}

Based on the tapped delay line model, a RAKE receiver (as in Fig. 4.3.1) [12] can be used to detect the signal from the multipath fading channel. Let us consider binary signaling over the channel. We have two equal-energy signal $u_{1}(t)$ and $u_{2}(t)$ which are 
either antipodal or orthogonal. Their time duration $T$ is selected to satisfy the condition $T \gg T_{m}$. Thus we may neglect any intersymbol interference due to multipath. Since the bandwidth of the signal exceeds the coherent bandwidth of the channel, the received signal is expressed as

$$
\begin{aligned}
r(t) & =\sum_{k=1}^{L} c_{k}(t) u_{i}\left(t-\frac{n}{W}\right)+z(t) \\
& =v_{i}(t)+z(t) \quad 0 \leq t \leq T \quad i=1,2
\end{aligned}
$$

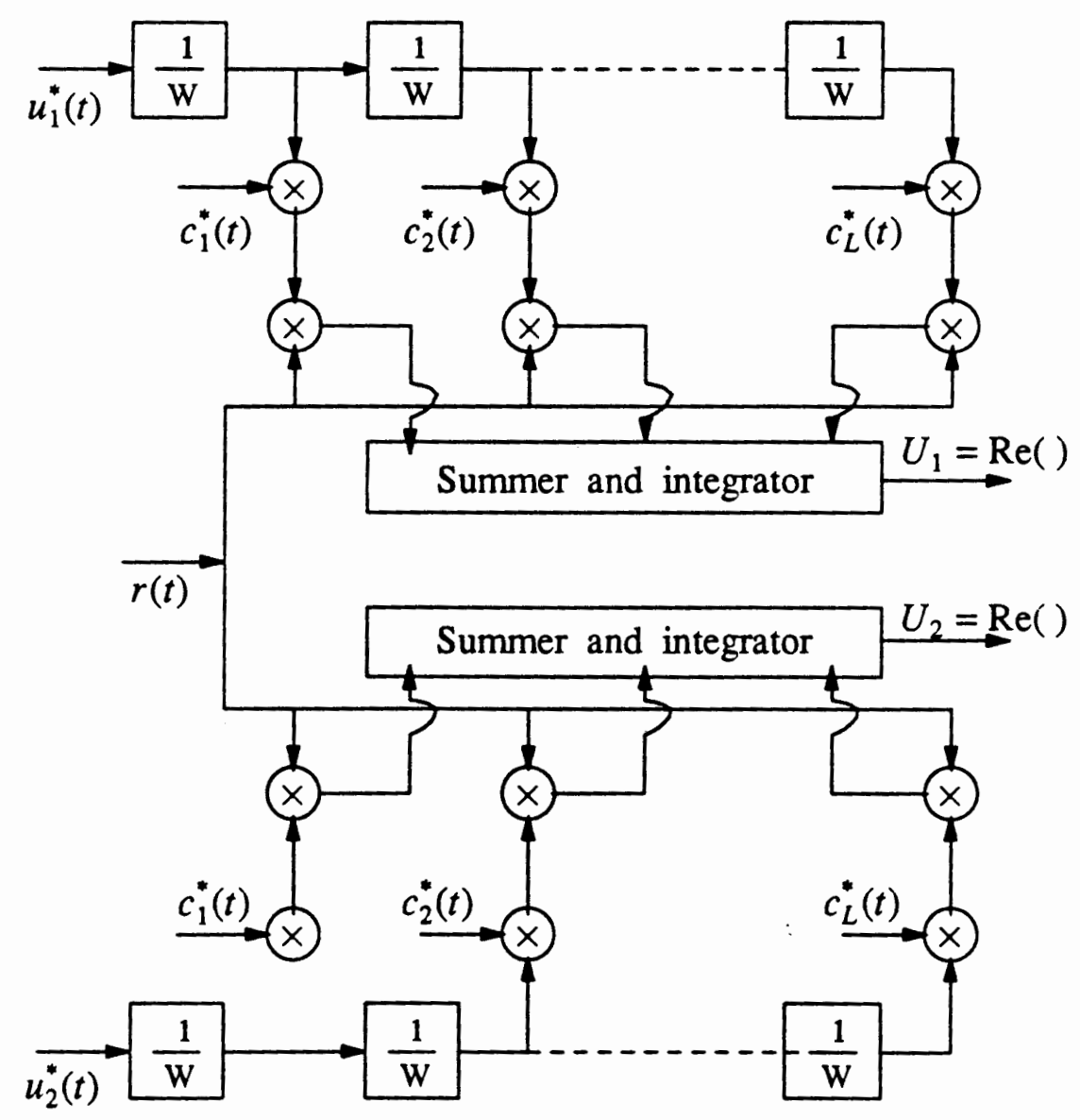

Fig. 4.3.1. Optimum demodulator for wideband signal 
where $z(t)$ is a complex-valued zero mean white gaussian noise process. Assume for the moment that the channel tap weights are known. Then the optimum receiver consists of two filters matched to $v_{1}(t)$ and $v_{2}(t)$, followed by samplers and a decision circuit that selects the signal corresponding to the largest output.

An equivalent optimum receiver employs cross correlation instead of matched filtering. In either case, the decision variables for coherent detection of the binary signals can be expressed as

$$
\begin{aligned}
U_{m} & =\operatorname{Re} \int_{0}^{T} r(t) v_{m}^{*}(t) d t \\
& =\operatorname{Re} \sum_{k=1}^{L} \int_{0}^{T} r(t) c_{k}^{*}(t) u_{m}^{*}\left(t-\frac{k}{W}\right) d t \quad m=1,2
\end{aligned}
$$

Fig. 4.3.1 illustrates the operations involved in the computation of the decision variables. In this realization of the optimum receiver, the two reference signals are delayed and correlated with the received signal $r(t)$.

An alternative realization of the optimum receiver employs a single delay line through which is passed the received signal $r(t)$. The signal at each tap is correlated with $c_{k}(t) u_{m}^{*}(t)$, where $k=1,2, \ldots, L$ and $m=1,2$. This receiver structure is shown in Fig. 4.3.2. In effect, the tapped delay line receiver attempts to collect the signal energy from all the received signal paths that fall within the span of the delay line and carry the same information. Its action is somewhat analogous to an ordinary garden rake and, consequently, the name $R A K E$ receiver has been coined for this receiver structure by Price and Green (1958) [10] .

The decision variables for the coherent detection of the binary signals in Eq.(4.3.2) may be expressed in the form 


$$
U_{m}=\operatorname{Re}\left[\sum_{k=1}^{L} c_{k}^{*} \int_{0}^{T} r(t) u_{m}^{*}\left(t-\frac{k}{W}\right) d t\right] \quad m=1,2
$$

If the transmitted signal is $u_{1}(t)$; then the received signal is

$$
r(t)=\sum_{n=1}^{L} c_{n} u_{1}\left(t-\frac{k}{W}\right) d t+z(t) \quad 0 \leq t \leq T
$$

Substitution of Eq.(4.3.4) into Eq.(4.3.3) gives

$$
\begin{aligned}
U_{m}= & \operatorname{Re}\left[\sum_{k=1}^{L} c_{k}^{*} \sum_{n=1}^{L} c_{n} \int_{0}^{T} u_{1}\left(t-\frac{n}{W}\right) u_{m}^{*}\left(t-\frac{k}{W}\right) d t\right] \\
& +\operatorname{Re}\left[\sum_{k=1}^{L} c_{k}^{*} \int_{0}^{T} z(t) u_{m}^{*}\left(t-\frac{k}{W}\right) d t\right] \quad m=1,2
\end{aligned}
$$

Since the wideband signals $u_{1}(t)$ and $u_{2}(t)$ are generated from pseudo-random sequences, which satisfy

$$
\int_{0}^{T} u_{i}\left(t-\frac{n}{W}\right) u_{i}^{*}\left(t-\frac{k}{W}\right) d t \approx 0 \quad \text { for } k \neq n, i=1,2
$$

then Eq.(4.3.5) is simplified as

$$
\begin{aligned}
U_{m}= & \operatorname{Re}\left[\sum_{k=1}^{L}\left|c_{k}\right|^{2} \int_{0}^{T} u_{1}\left(t-\frac{k}{W}\right) u_{m}^{*}\left(t-\frac{k}{W}\right) d t\right] \\
& +\operatorname{Re}\left[\sum_{k=1}^{L} c_{k}^{*} \int_{0}^{T} z(t) u_{m}^{*}\left(t-\frac{k}{W}\right) d t\right] \quad m=1,2
\end{aligned}
$$


When the binary signals are antipodal, a signal decision variable suffices. In this case Eq.(4.3.7) reduces to

$$
U_{1}=2 \varepsilon \sum_{k=1}^{L}\left|c_{k}\right|^{2}+\operatorname{Re}\left[\sum_{k=1}^{L} c_{k}^{*} \int_{0}^{T} z(t) u_{m}^{*}\left(t-\frac{k}{W}\right) d t\right]
$$

But Eq.(4.3.8) is identical to the decision variable, which corresponds to the output of a maximal ratio combiner in a system with $L$ th-order diversity. Consequently the $R A K E$ receiver with perfect (noiseless) estimates of the channel tap weights is equivalent to a maximal ratio combiner in a system with $L$ th-order diversity.

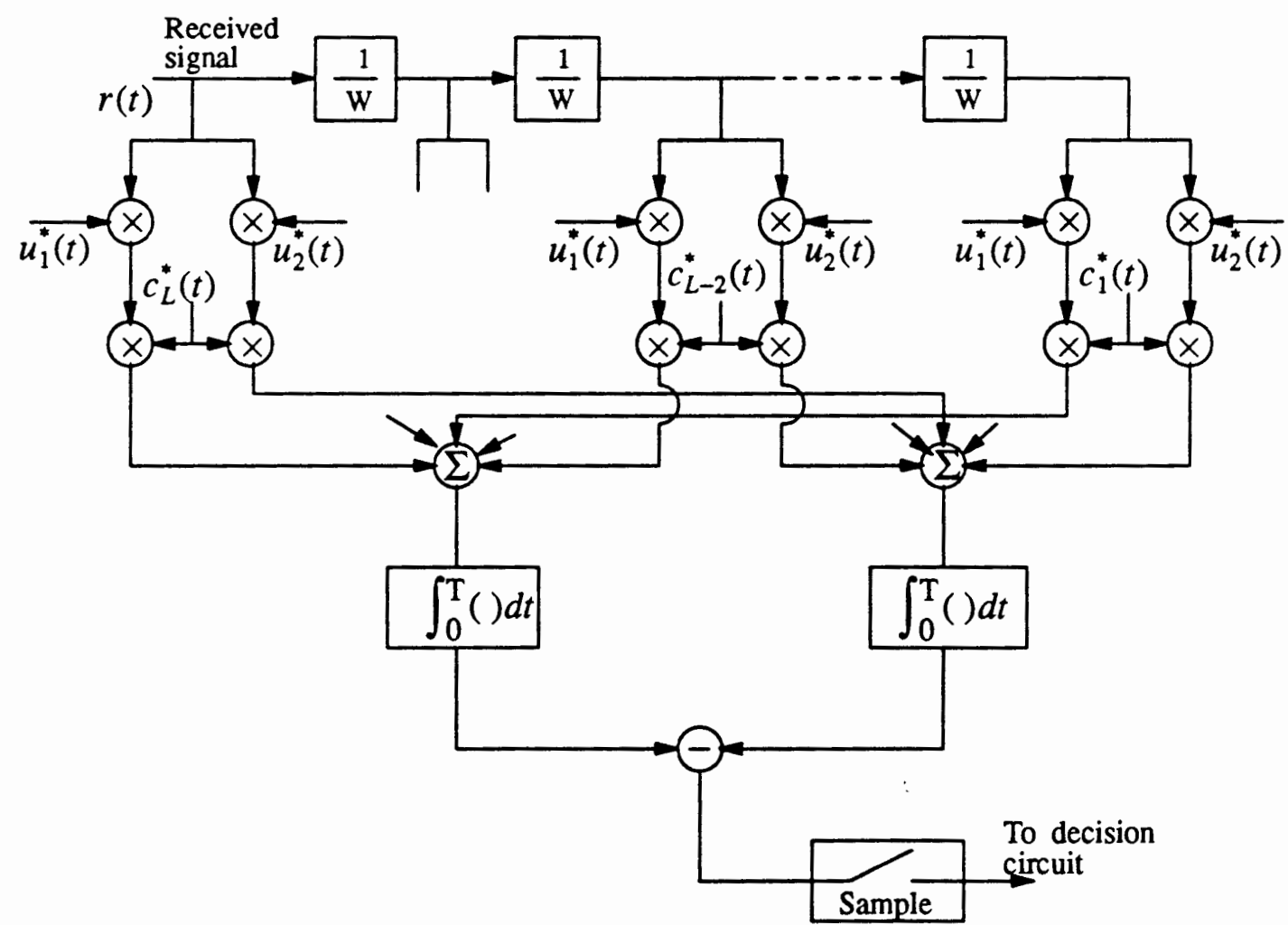

Fig. 4.3.2. Optimum demodulator for delay received signal configuration 
Thus, when all the tap weights have the same mean-square value, i.e., $E\left(\left|c_{k}\right|^{2}\right)$ is the same for all $k$, the error rate performance of the RAKE receiver is given by Eq.(4.3.9) and Eq.(4.3.10).

$$
P_{2}=\left(\frac{1-\mu}{2}\right)^{L} \sum_{k=0}^{L-1}\left(\begin{array}{c}
L-1+k \\
k
\end{array}\right)\left(\frac{1+\mu}{2}\right)^{k}
$$

where, by definition,

$$
\mu=\sqrt{\frac{\gamma_{c}}{1+\gamma_{c}}}
$$

where $\gamma_{c}$ is the average SNR per channel, which is assumed to be identical for all channels. That is,

$$
\gamma_{c}=\frac{\mathcal{E}}{N_{0}} E\left(\left|c_{k}\right|^{2}\right)
$$

independent of $k$.

$N_{0}$ is the power spectral density of $z(t)$, where variance of $z(t)=N_{0} / 2$ and

$$
\varepsilon=\frac{1}{2} \int_{0}^{T}\left|u_{m}(t)\right|^{2} d t \quad m=1,2, \ldots, L
$$

On the other hand, when the mean-square value $E\left(\left|c_{k}\right|^{2}\right)$ are not identical for all $k$, the derivation of the error rate performance must be repeated since Eq.(4.3.9) no longer applies.

When the mean-square values of the tap weights are distinct, the error rate 
performance of the $R A K E$ receiver is given by Eq.(4.3.13)

$$
P_{2}=\frac{1}{2} \sum_{k=1}^{L} \pi_{k}\left[1-\sqrt{\frac{\gamma_{k}\left(1-\rho_{r}\right)}{2+\gamma_{k}\left(1-\rho_{r}\right)}}\right]
$$

This error probability can be approximated as $\left(\gamma_{k} \gg 1\right)$

$$
P_{2} \approx\left(\begin{array}{c}
2 L-1 \\
L
\end{array}\right) \prod_{k=1}^{L} \frac{1}{\left[2 \gamma_{k}\left(1-\rho_{r}\right)\right]}
$$

where $\rho_{r}=-1$ for antipodal signals, $\rho_{r}=0$ for orthogonal signals, and

$$
\gamma_{k}=\frac{\varepsilon}{N_{0}} E\left(\left|c_{k}\right|^{2}\right)
$$

and $\pi_{k}$ is defined as

$$
\pi_{k}=\prod_{\substack{i=1 \\ i \neq k}}^{L} \frac{\gamma_{k}}{\gamma_{k}-\gamma_{i}}
$$

\section{IV.4 CONCLUSION}

In the above analysis, we discuss how a $R A K E$ receivcy ian be used to detect the signal from the multipath fading channel and how a $R A K E$ receiver can work against the combination of random multipath and additive noise disturbances. Finally, we evaluate the performance of the $R A K E$ receiver under the condition that the fading is sufficiently slow. 
In a $R A K E$ receiver, if the tap weights are correlated, the error rate performance of the $R A K E$ receiver will decrease. The limitation of $R A K E$ receiver is that it doesn't work perfectly for correlated tap weights. 


\section{CHAPTER V}

\section{DETECTION : AIC and MDL APPROACH}

\section{V.1 INTRODUCTION}

In the derivation of RAKE receiver [12], the number of delay paths (or taps), the time delay of each path and the channel tap weights are assumed known at receiver. However, to practically implement $R A K E$ receiver, the number of delay paths (or taps) $\{L\}$, the time delay of each path $\{\tau\}$ and the tap weights $\left\{c_{n}(t)\right\}$ need to be estimated. After those, a diversity receiver ( $R A K E$ receiver) can be used to detect the signal from the the multipath fading channel.

To estimate the tap weights after assumed the number of delay paths (or taps) and the time delay of each path known at receiver, earlier work [12] employed a single correlator. Its output is fed to the input of the low-pass filter after information-bearing signal is removed. To accomplish this, a delay of one signaling interval is introduced into the channel estimation procedure, as illustrated in Fig. 5.1.1. That is, first the ir.siver must decide whether the information in the received signal is +1 or -1 and, then, it uses the decision to remove the information from the correlator output prior to feeding it to the low-pass filter.

The objective of the following three chapters is to explore the possibility of using the advanced signal processing algorithms to detect the number of delay paths $\{L\}$, to estimate the time delay of each path $\{\tau\}$ and to estimate the tap weight for each delay 
path in multipath channels, respectively [39] [46] .

Contributions included in this chapter are [39] :

1) A general description of $I S-95$ standard.

2) A general description of pilot channel based on pilot PN chip rate.

3) A general description of bit-error-rate (BER) and frame-error-rate (FER).

4) An "Information Theoretic Criterion" detection approach (AIC and MDL as described in [40]) to determine the number of delay path.

5) Simulation results on bit-error-rate (BER) as a function of $E_{b} / N_{0}$ using BPSK modulation for the multipath fading channel.

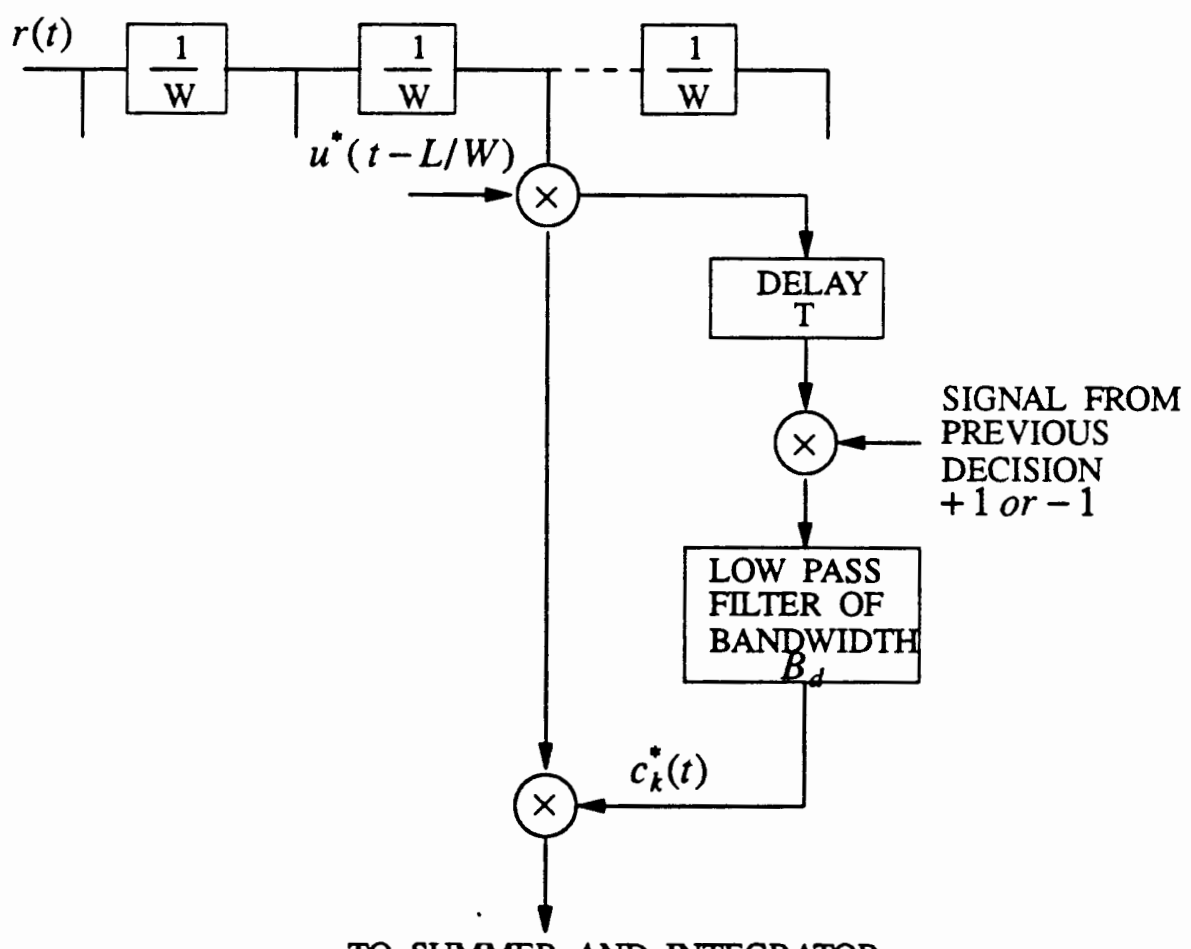

TO SUMMER AND INTEGRATOR

Fig. 5.1.1. Tap Weight Estimation 


\section{V.2 IS-95 STANDARD}

IS-95 is a $900 \mathrm{MHz}$ CDMA cellular communications standard that has been adopted in some US and foreign markets. The standard will also be used in the 1900 MHz PCS band (PCS - Personal Communication Service) .

For a IS-95 model (CDMA) (Fig. 5.2.1)

Table 5.2.1 : Rayleigh distribution

\begin{tabular}{|c|c|c|}
\hline Taps & Rel Delay $(\mu \mathrm{Sec})$ & Ave Power $(\mathrm{dB})$ \\
\hline 1 & 0 & 0 \\
\hline 2 & 2 & 0 \\
\hline 3 & 14.5 & -3 \\
\hline
\end{tabular}

In the table, Relative Delay is relative to the first path. Average power attenuation is also relative to the first path. In Fig. 5.2.1, $\tau_{1}=0 ; \tau_{2}=2 \mu s ; \tau_{2}=14.5 \mu \mathrm{s}$.

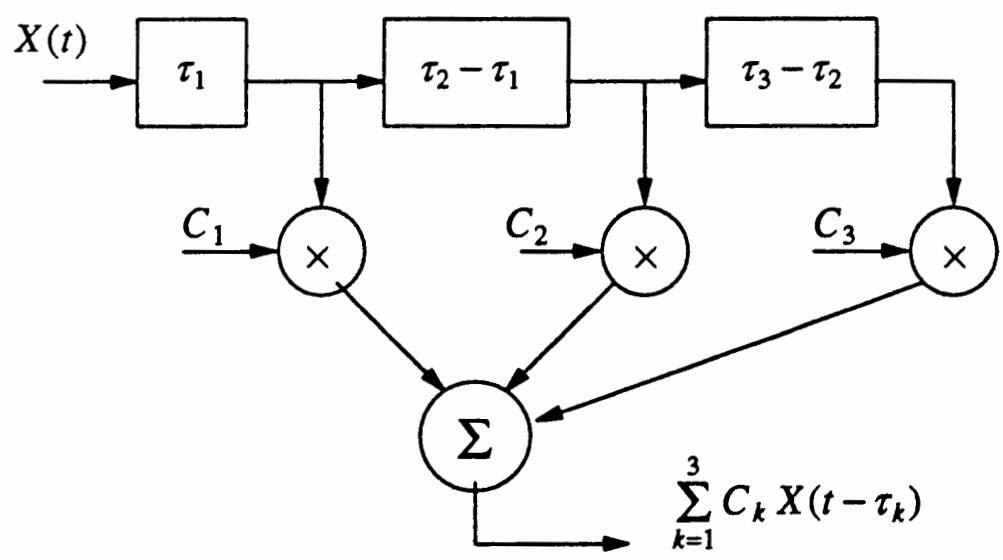

Fig. 5.2.1. IS-95 standard

The Telecommunications Industry Association IS-95 standard (called TIA IS-95) uses a 64 by 64 Walsh matrix to generate 64 mutually orthogonal waveforms. In the 
forward channel, the Walsh function is used for orthogonal spread. A different channel is assigned to a different Walsh function to provide the orthogonality of transmitted channel information from the same sector/cell.

\section{V.3 PILOT CHANNEL}

A pilot channel is transmitted at all times by the base station on each active forward CDMA channel. The pilot channel is an unmodulated spread spectrum signal that is used for synchronization by a mobile station operating within the coverage area of the base station.

Each base station shall use a time offset of the pilot PN sequence to identify a forward CDMA channel. Time offsets may be reused within a CDMA cellular system.

Pseudorandom noise (PN) binary codes are used to distinguish signals from different base stations received at a mobile station. All CDMA signal in the system share a quadrature pair of PN codes. Signals from different cells and sectors are distinguished by time offsets from the basic code. This relies on the property of PN codes that the auto-correlation (when averaged over a few bit times) has an average which approaches zero for all time offsets greater than a single code chip time (approximatedly $1 \mu \mathrm{s}$ ). The PN codes used are generated by linear shift registers that produce a code with a period of 32768 chips $\left(2^{15}=32768\right.$ i.e., 15 stages). The PN chips rate is $1.2288 \mathrm{MHz}$, or exactly 128 times the 9600 bps information transmission rate. Two codes are generated, one for each quadrature carrier, which results in quadra-phase PN modulation.

To avoid confusion between the system bandwidth, the PN chip rate, and the frequency assignment spacing, note that the PN chip rate is exactly $1.2288 \mathrm{MHz}$.

All signals transmitted from a cell in a particular CDMA radio channel share a common PN code phase. Orthogonality provides nearly perfect isolation between the 
multiple signals transmitted by the base station.

Distinct pilot channels shall be identified by an offset index ( 0 through 511 inclusive). This offset index specifies the offset value from the zero offset pilot PN sequence. The zero offset pilot PN sequence shall be such that the start of the sequence shall be output at the beginning of every even second in time, referenced to base station transmission time.

Five hundred and twelve unique values are possible for the pilot PN sequence offset. The offset (in chips) for a given pilot PN sequence from the zero shift pilot PN sequence equals the index value multiplied by 64. For example, if the pilot PN sequence offset index is 15, the pilot PN sequence offset will be $15 \times 64=960$ PN chips. In this case the pilot PN sequence will start $781.25 \mu$ s after the start of every even second of time, referenced to base station transmission time. The same pilot PN sequence offset shall be used on all CDMA frequency assignments for a given base station.

Prior to transmission, the pilot channel shall be spread with Walsh function.

For mobile system, rapid fading is a central problem in digital mobile communications. It degrades the bit error rate (BER), and frequently introduces an irreducible BER, or error floor. The use of a pilot tone to mitigate the effects of fading has been explored by several authors [30] - [33]. The tone provides the receiver with an explicit amplitude and phase reference for detection, and thereby suppresses the error floor. The question of where in the spectrum to locate the tone is a difficult one.

Recently, pilot symbol assisted modulation (PSAM) has been proposed [34] - [36] as an alternative. The transmitter periodically insert known symbols, from which the receiver derives its amplitude and phase reference. Like pilot tone modulation, PSAM suppresses the error floor and enables multilevel modulation. However, it does so with no change to the transmitted pulse shape or peak to average power ratio. Although they 
demonstrated feasibility, they did not provide the performance analysis needed before their results can be generalized.

The study of the performance of the optimized system has been explored by [38]. However, the channel tap weights are assumed known at receiver in [38] .

\section{V.4 BIT-ERROR-RATE ( BER)}

System performance is evaluated by Bit-Error-Rate (BER) and Frame-Error-Rate (FER) as a function of $E_{b} / N_{0}$, the ratio of the combined received energy per-bit to the effective noise power spectral density for the sync channel, paging channel, or traffic channel at the mobile station antenna.

The BER is calculated through bit which is defined as:

$$
B E R=\frac{\text { number of error bits }}{\text { number of total transmitted bits }} .
$$

The FER is calculated through block which is defined as:

$$
F E R=\frac{\text { number of error frames }}{\text { number of total transmitted frames }} .
$$

where error frame is defined as the frame that CRC check fails.

Two sets of simulation are carried out using the Monte Carlo method. The number of frames chosen for simulation is corresponding to $95 \%$ confidence interval. That is $10^{2+1}$ i.e., 1000 frames for FER to achieve $10^{-2}$. 


\section{V.5 MULTIPATH FADING CHANNEL}

Multipath fading channel is usually modeled as a time-variant tapped delay system (see Fig. 5.5.1).

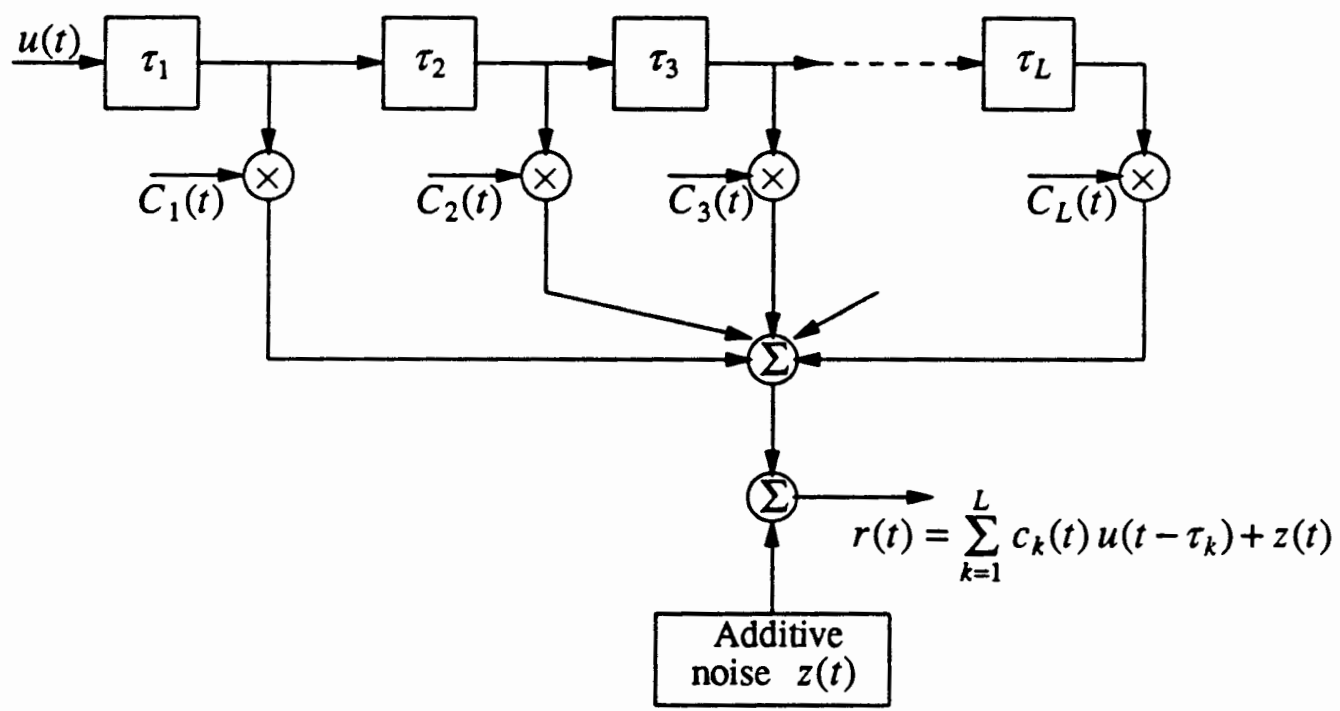

Fig. 5.5.1. Tapped Delay Model for Multinnth Fading.

The signal arrived at receiver can be expressed as

$$
\begin{aligned}
& r(t)=\sum_{n=1}^{L} c_{n}(t) u\left(t-\tau_{n}\right)+z(t)= \\
& =\left[\begin{array}{lll}
u\left(t-\tau_{1}\right) & \cdots & u\left(t-\tau_{L}\right)
\end{array}\right]\left[\begin{array}{c}
c_{1}(t) \\
\cdot \\
\cdot \\
\cdot \\
c_{L}(t)
\end{array}\right]+z(t)
\end{aligned}
$$


where $\tau_{n}$ is the time-delay at each path, $z(t)$ is the additive channel noise, and $u(t)$ is the signal transmitted through the multipath fading channel. The time-variant tap weights $\left\{c_{n}(t)\right\}$ are zero-mean complex-valued stationary processes statistically independent to each other [12], but for a slowly fading channel, $\left\{c_{n}(t)\right\}$ is a constant within a chip. Therefore, if we sample the received data $K$ times, we have

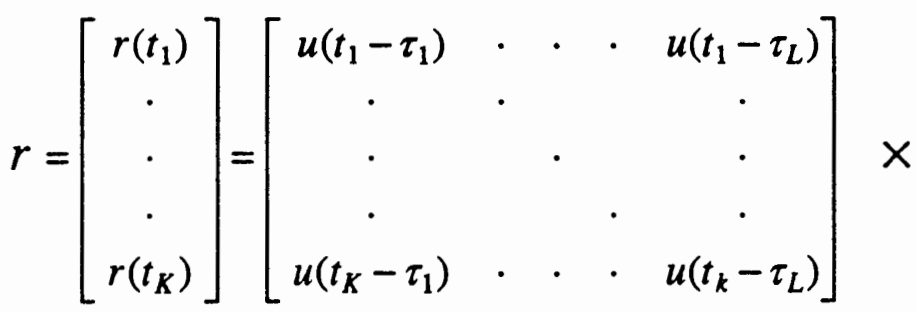

$$
\begin{aligned}
& {\left[\begin{array}{c}
c_{1} \\
\cdot \\
\cdot \\
c_{L}
\end{array}\right]+\left[\begin{array}{c}
z\left(t_{1}\right) \\
\cdot \\
\cdot \\
\cdot \\
z\left(t_{K}\right)
\end{array}\right]}
\end{aligned}
$$

We define the vector $u(\tau)$

$$
u(\tau)=\left[\begin{array}{lll}
u\left(t_{1}-\tau\right) & \cdots & u\left(t_{K}-\tau\right)
\end{array}\right]^{H}
$$

for future use. Notice that $u(\tau)$ has a known form (known waveform in communication) and unknown delay $\tau$. Further, if same signal could be repeated $N$ times (as signals in pilot channel, for example), $c_{n}$ will be different each time, then we can form a data matrix

$$
R=\left[\begin{array}{lll}
r_{1} & \cdots & r_{N}
\end{array}\right]
$$

where each $r_{j}$ has a same form as in Eq.(5.5.2) except $c_{n}$ is different. In matrix form 


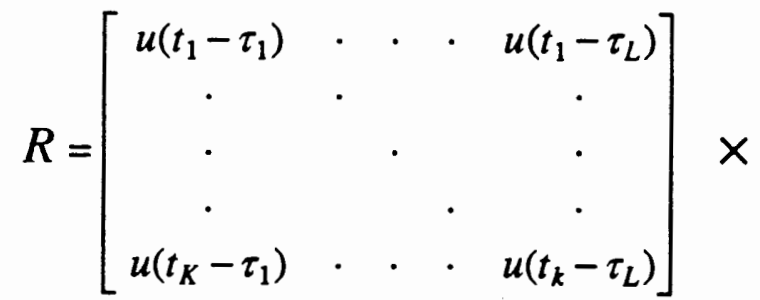

$$
\begin{aligned}
& {\left[\begin{array}{cccc}
c_{11} & \cdot & \cdot & \cdot c_{1 N} \\
\cdot & \cdot & & \cdot \\
\cdot & & \cdot & \cdot \\
\cdot & & & \cdot \\
c_{L 1} & \cdot & \cdot & \cdot \\
c_{L N}
\end{array}\right]+\left[\begin{array}{cccc}
z_{1}\left(t_{1}\right) & \cdot & \cdot & \cdot z_{N}\left(t_{1}\right) \\
\cdot & \cdot & \cdot \\
\cdot & & \cdot & \cdot \\
\cdot & & \cdot & \cdot \\
z_{1}\left(t_{K}\right) & \cdot & \cdot & \cdot z_{N}\left(t_{K}\right)
\end{array}\right]}
\end{aligned}
$$

or equivalently, in vector notation

$$
R=U(\tau) C+Z
$$

The sample covariance matrix is

$$
\operatorname{Cov}=\frac{1}{N} R R^{H}
$$

where superscript $H$ stands for Hermitian conjugate. An eigenvalue (or singular value) decomposition can be performed one the sample covariance matrix

$$
\operatorname{Cov}=E \Lambda E^{H}
$$

where $E$ and $\Lambda$ are eigenvalue and eigenvector matrices, respectively.

\section{V.6 DETECTION : AIC and MDL APPROACH}

An information theoretic criterion that Akaike [41] developed a method, called Akaike information criterion (AIC), for parametric model fitting to observed data. Based 
on the point of view of the minimum (shortest) data description (MDL), Rissanen [42] also developed a similar method. The direct application of these techniques to the problem of determining the number of sources with the model given in $[41,42]$ would be very difficult, mainly due to the complicated nature of likelihood maximization over all the parameters specifying the model.

Wax and Kailath [43] applied AIC and MDL methods to the problem of determining the number of sources. A pre-overparameterization of the model in term of the eigenvalues and eigenvectors of $R$ was used to deduce the computational complexity of the methods.

Let eigenvalues $\lambda_{i}(i=1, \ldots, K)$ be decreasingly ordered on the diagonal of $\Lambda$. We can apply information theoretic criterion to determine the number of delay paths, that is to estimate $\hat{L}$ by minimizing the following function over the possible nuriciver of delay paths $l$.

$$
\Phi(l, N)=N(K-l) \log \left[\frac{a(l)}{q(l)}\right]+\phi(l, N)
$$

where

$$
\begin{aligned}
& a(l)=\frac{1}{K-l} \sum_{i=l+1}^{K} \lambda_{i} \\
& q(l)=\left(\prod_{i=l+1}^{K} \lambda_{i}\right)^{\frac{1}{K-l}}
\end{aligned}
$$

where $\phi(l, N)$ is a penalty function for the overdetermination of the number of delay paths with the overparametrized model and log denotes the natural logarithm. The function is given by 


$$
\phi(l, N)=l(2 K-l)
$$

for the AIC method and

$$
\phi(l, N)=\frac{1}{2} l(2 K-l) \log N
$$

for the MDL method.

It is easy to see that the MDL method places a heavier penalty on the overdetermination of the number of delay paths.

Using AIC and MDL methods, we can estimate the number of delay paths $\hat{L}$, by minimizing the Eq.(5.6.1) over $l$.

\section{V.7 PERFORMANCE COMPARISON}

In our computer simulations, we choose three paths with delays $\tau=1,7,20(T)$, respectively. The ratio of amplitude attenuation of the second path over that of first path is $-3 \mathrm{~dB}$, and of third path over the first path is $-6 \mathrm{~dB}$.

Those equations are :

$$
\begin{aligned}
& 20 \log \left[\frac{\left|C_{2}\right|}{\left|C_{1}\right|}\right]=-3 d B \\
& 20 \log \left[\frac{\left|C_{3}\right|}{\left|C_{1}\right|}\right]=-6 d B
\end{aligned}
$$

The fundamental frequency of the binary message signal $m(t)$ is chosen to be 19.2 KHz, and the fundamental frequency of PN code sequence $b(t)$ is 
$64 \times 19.2 \mathrm{KHz}=1.2288 \mathrm{MHz}$.

Fig. 5.7.1 shows the detection probability (for correctly estimate the number of delay paths) versus energy-per-bit over noise-density $\left(E_{b} / N_{0}\right)$ using both AIC and MDL detection algorithms. From the Fig. 5.7.1, We can know the correct detection probability of the number of delay paths is equal to 1 after $E_{b} / N_{0}=6.5 d B$ for both AIC and MDL detection algorithms.

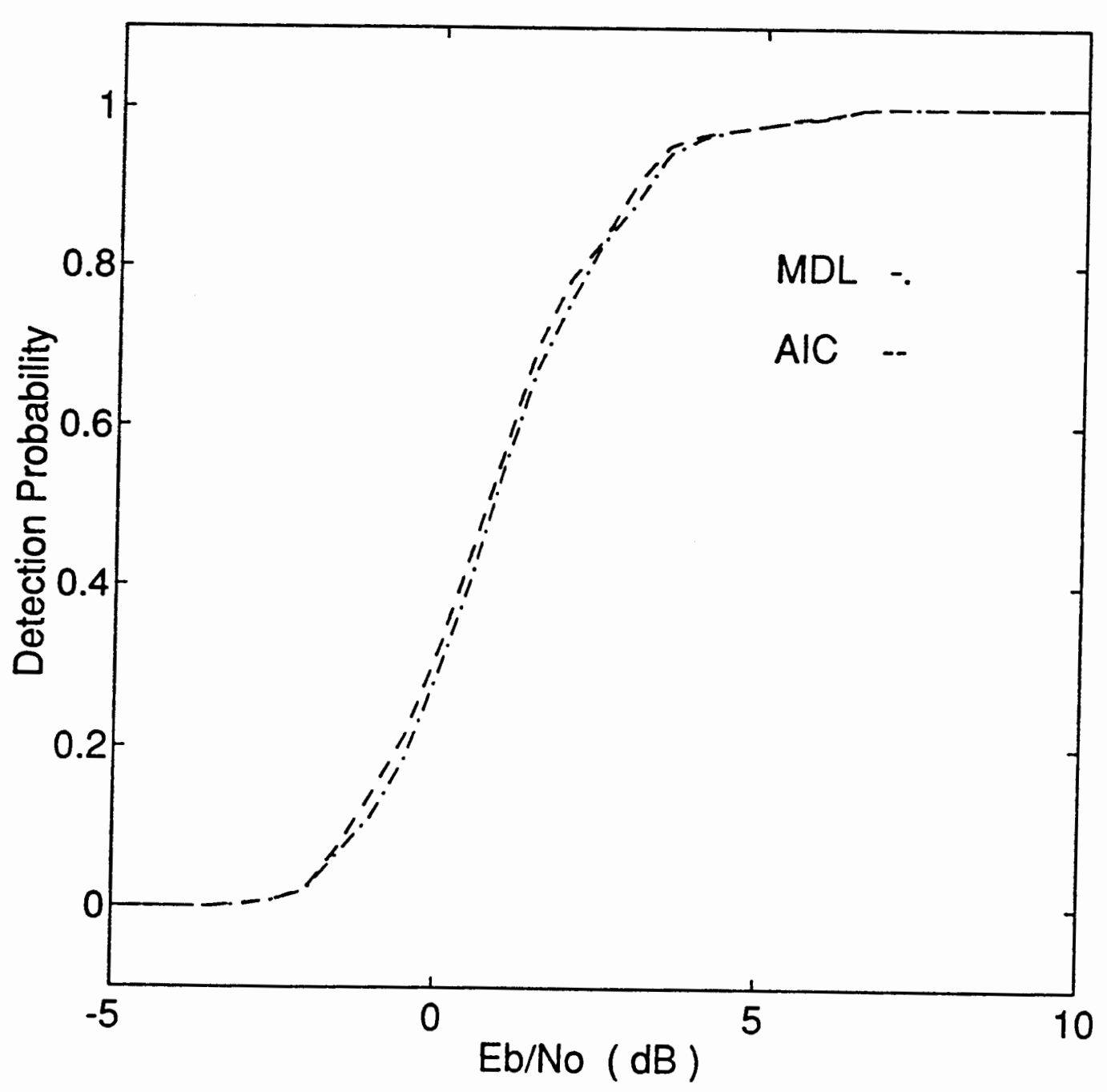

Fig. 5.7.1. Delay Detection Probability verse $E_{b} / N_{0}$ 


\section{V.8 CONCLUSION}

In this chapter, we explore the possibility of using the advanced signal processing algorithms (using AIC and MDL methods) to detect the number of delay paths $\{L\}$. Clearly, those methods have better performance to detect the number of delay paths in a realistic mobile environment. 


\section{CHAPTER VI}

\section{ESTIMATION : MUSIC AND MIN-NORM APPROACH}

\section{VI.1 INTRODUCTION}

In chapter $\mathrm{V}$, we detect the number of delay paths (or taps). However, to practically implement $R A K E$ receiver, the time delay of each path $\{\tau\}$ need to be estimated. After that, a diversity receiver ( $R A K E$ receiver) can be used to detect the signal from the multipath fading channel.

The objective of the chapter is to explore the possibility of using the advanced signal processing algorithms to estimate the time delay of each path $\{\tau\}$ in a realistic mobile environment [46].

Contributions included in this chapter are [46] :

1) A general description of diversity receiver based on chip rate channel estimate.

2) A subspace-based estimation approach (MUSIC [44] and Min-Norm [45]) to determine the time delay of each path.

3) Simulation results on bit-error-rate (BER) as a function of $E_{b} / N_{0}$ using BPSK modulation for the multipath fading channel. 


\section{VI.2 MULTIPATH FADING CHANNEL}

Multipath fading channel is usually modeled as a time-variant tapped delay system (see Fig. 6.2.1).

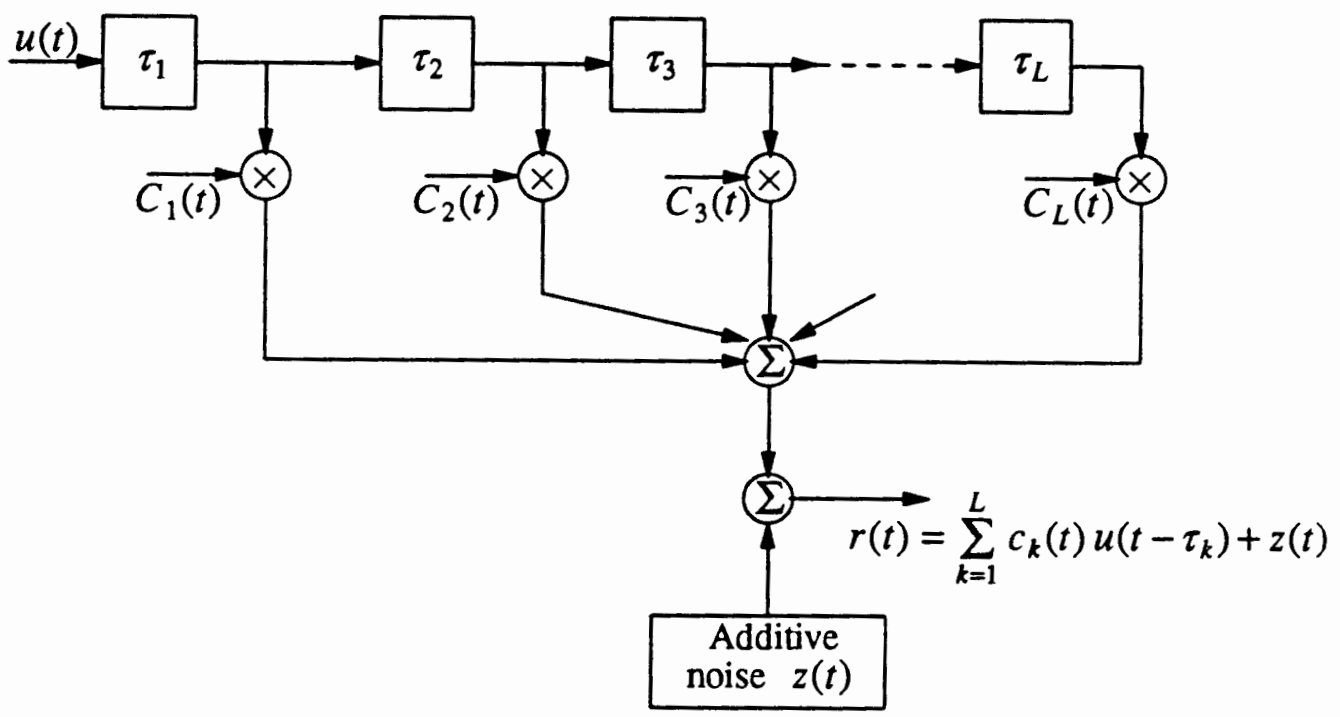

Fig. 6.2.1. Tapped Delay Model for Multipath Fading.

The signal arrived at receiver can be expressed as

$$
\begin{aligned}
& r(t)=\sum_{n=1}^{L} c_{n}(t) u\left(t-\tau_{n}\right)+z(t)= \\
& =\left[\begin{array}{lll}
u\left(t-\tau_{1}\right) & \cdots & u\left(t-\tau_{L}\right)
\end{array}\right]\left[\begin{array}{c}
c_{1}(t) \\
\cdot \\
\cdot \\
\cdot \\
c_{L}(t)
\end{array}\right]+z(t)
\end{aligned}
$$


where $\tau_{n}$ is the time-delay at each path, $z(t)$ is the additive channel noise, and $u(t)$ is the signal transmitted through the multipath fading channel. The time-variant tap weights $\left\{c_{n}(t)\right\}$ are zero-mean complex-valued stationary processes statistically independent to each other [12], but for a slowly fading channel, $\left\{c_{n}(t)\right\}$ is a constant within a chip. Therefore, if we sample the received data $K$ times, we have

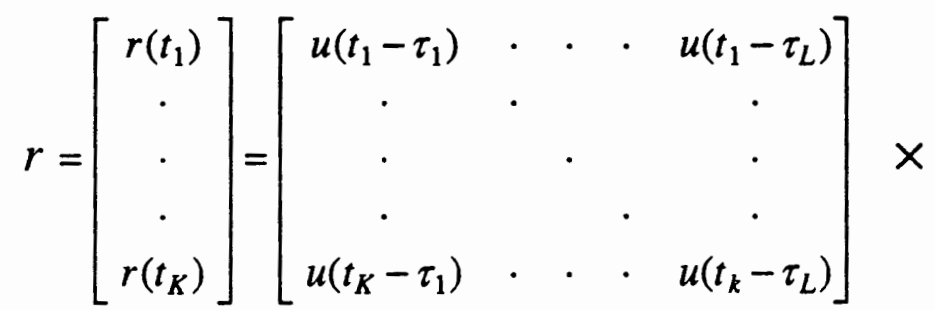

$$
\begin{aligned}
& {\left[\begin{array}{c}
c_{1} \\
\cdot \\
\cdot \\
c_{L}
\end{array}\right]+\left[\begin{array}{c}
z\left(t_{1}\right) \\
\cdot \\
\cdot \\
\cdot \\
z\left(t_{K}\right)
\end{array}\right]}
\end{aligned}
$$

We define the vector $u(\tau)$

$$
u(\tau)=\left[\begin{array}{lll}
u\left(t_{1}-\tau\right) & \cdots & u\left(t_{K}-\tau\right)
\end{array}\right]^{H}
$$

for future use. Notice that $u(\tau)$ has a known form (known waveform in communication) and unknown delay $\tau$. Further, if same signal could be repeated $N$ tirites (as signals in pilot channel, for example), $c_{n}$ will be different each time, then we can form a data matrix

$$
R=\left[\begin{array}{lll}
r_{1} & \cdots & r_{N}
\end{array}\right]
$$

where each $r_{j}$ has a same form as in Eq.(6.2.2) except $c_{n}$ is different. In matrix form 


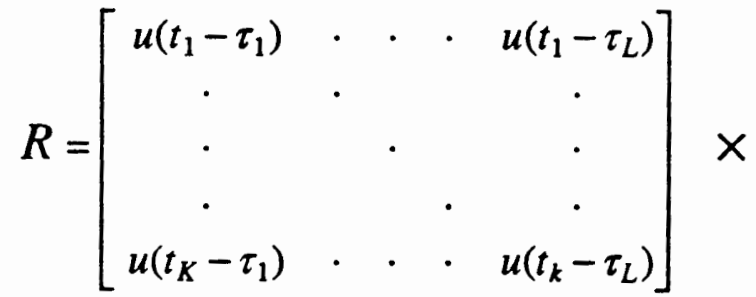

$$
\begin{aligned}
& {\left[\begin{array}{cccc}
c_{11} & \cdot & \cdot & \cdot c_{1 N} \\
\cdot & \cdot & & \cdot \\
\cdot & & \cdot & \cdot \\
\cdot & & & \cdot \\
c_{L 1} & \cdot & \cdot & \cdot \\
c_{L N}
\end{array}\right]+\left[\begin{array}{cccc}
z_{1}\left(t_{1}\right) & \cdot & \cdot & \cdot z_{N}\left(t_{1}\right) \\
\cdot & \cdot & & \cdot \\
\cdot & & \cdot & \cdot \\
\cdot & & \cdot & \cdot \\
z_{1}\left(t_{K}\right) & \cdot & \cdot & \cdot z_{N}\left(t_{K}\right)
\end{array}\right]}
\end{aligned}
$$

or equivalently, in vector notation

$$
R=U(\tau) C+Z
$$

The sample covariance matrix is

$$
\operatorname{Cov}=\frac{1}{N} R R^{H}
$$

where superscript $H$ stands for Hermitian conjugate. An eigenvalue (or singular value) decomposition can be performed one the sample covariance matrix

$$
\operatorname{Cov}=E \Lambda E^{H}
$$

where $E$ and $\Lambda$ are eigenvalue and eigenvector matrices, respectively.

\section{VI.3 ESTIMATION : MI ${ }^{-3}$ IC AND MIN-NORM APPROACH}

The eigenvector matrix $E$ can be partitioned into 


$$
E=\left[E_{s} \mid E_{0}\right]
$$

where eigenvectors in $E_{s}$ are associated with $L$ (or $\hat{L}$ ) largest eigenvalues $\lambda_{i}(i=1, \ldots, L)$ and corresponding eigenvectors in $E_{0}$ are associated with $K-L$ smallested eigenvalues $\lambda_{i}(i=L+1, \ldots, K)$. From subspace theory, we can see $E_{s}$ is in the column span of $U(\tau)$ while $E_{0}$ is orthogonal to the column span of $U(\tau)$, i.e. $U(\tau)^{H} E_{0}=0$. We can then use the subspace algorithms to estimation the delay $\tau_{i}$.

For MUSIC ( MUltiple SIgnal Classification ) [44], we search for minimum of

$$
P(\tau)=\left|U(\tau)^{H} E_{0}\right|^{2}
$$

over $\tau$.

For Min-Norm [45], we search for minimum of

$$
P(\tau)=\left|u(\tau)^{H} d\right|^{2}
$$

over $\tau$. The linear prediction error vector

$$
d=E_{0} \frac{e_{1}}{\left\|e_{1}\right\|^{2}}
$$

where $e_{1}^{H}$ is the first row of $E_{0}$.

Using MUSIC or Min-Norm methods, we can estimate the time delay of each path by minimizing the Eq.(6.3.2) or Eq.(6.3.3) over $\tau$.

\section{VI.4 PERFORMANCE COMPARISON}

In our computer simulations, we choose three paths with delays $\tau=1,7,20(T)$, respectively. The ratio of amplitude attenuation of the second path over that of first path 
is $-3 \mathrm{~dB}$, and of third path over the first path is $-6 \mathrm{~dB}$.

Those equations are :

$$
\begin{aligned}
& 20 \log \left[\frac{\left|C_{2}\right|}{\left|C_{1}\right|}\right]=-3 d B \\
& 20 \log \left[\frac{\left|C_{3}\right|}{\left|C_{1}\right|}\right]=-6 d B
\end{aligned}
$$

The fundamental frequency of the binary message signal $m(t)$ is chosen to be 19.2 KHz, and the fundamental frequency of PN code sequence $b(t)$ is $64 \times 19.2 \mathrm{KHz}=1.2288 \mathrm{MHz}$. 
From the Fig. 5.7.1, we consider the result of time delay of each path when $E_{b} / N_{0} \geq 6.5 \mathrm{~dB}$. Fig. 6.4 .1 shows the delay spectrum $P(\tau)$ versus time at $E_{b} / N_{0}=10 d B$ using MUSIC algorithm. From the Fig. 6.4.1, We can know delay values

delay $\tau_{1}=64$ (samples) $=64 / 64$ (chips) $=1$ chip,

delay $\tau_{2}=448$ (samples) $=448 / 64$ (chips) $=7$ chips,

delay $\tau_{3}=1280$ (samples) $=1280 / 64$ (chips) $=20$ chips.

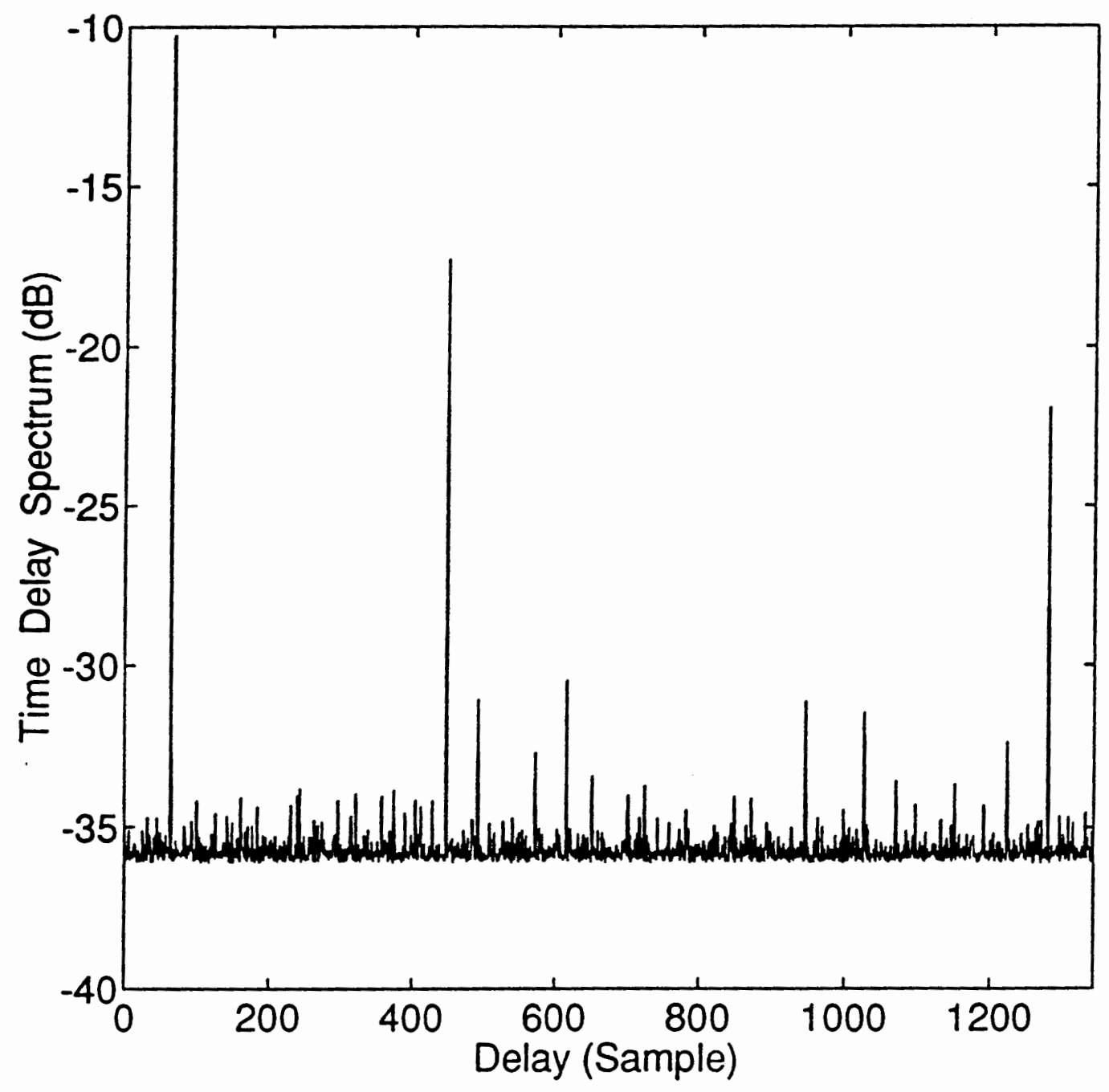

Fig. 6.4.1. Delay Spectrum at $E_{b} / N_{0}=10 d B$ 
Based on the result of Fig. 6.4.1, we compare time delay of each path which is obtained from Fig. 6.4.1 with correct time delay. Fig. 6.4.2 shows the root-meansquared error (RMSE) of the delay estimation for all three paths verse $E_{b} / N_{0}$.

From the Fig. 6.4.2, we can know $R M S E=0$ for delay 2 when $E_{b} / N_{0} \geq 0 d B$ and $R M S E=0$ for delay 3 when $E_{b} / N_{0} \geq 5 d B$. Because channel 3 receives deeper fading signal than channel 2 does, it is more difficult to estimate the time delay of channel 3 than the time delay of channel 2. Fig. 6.4.2 explores this kind of character.

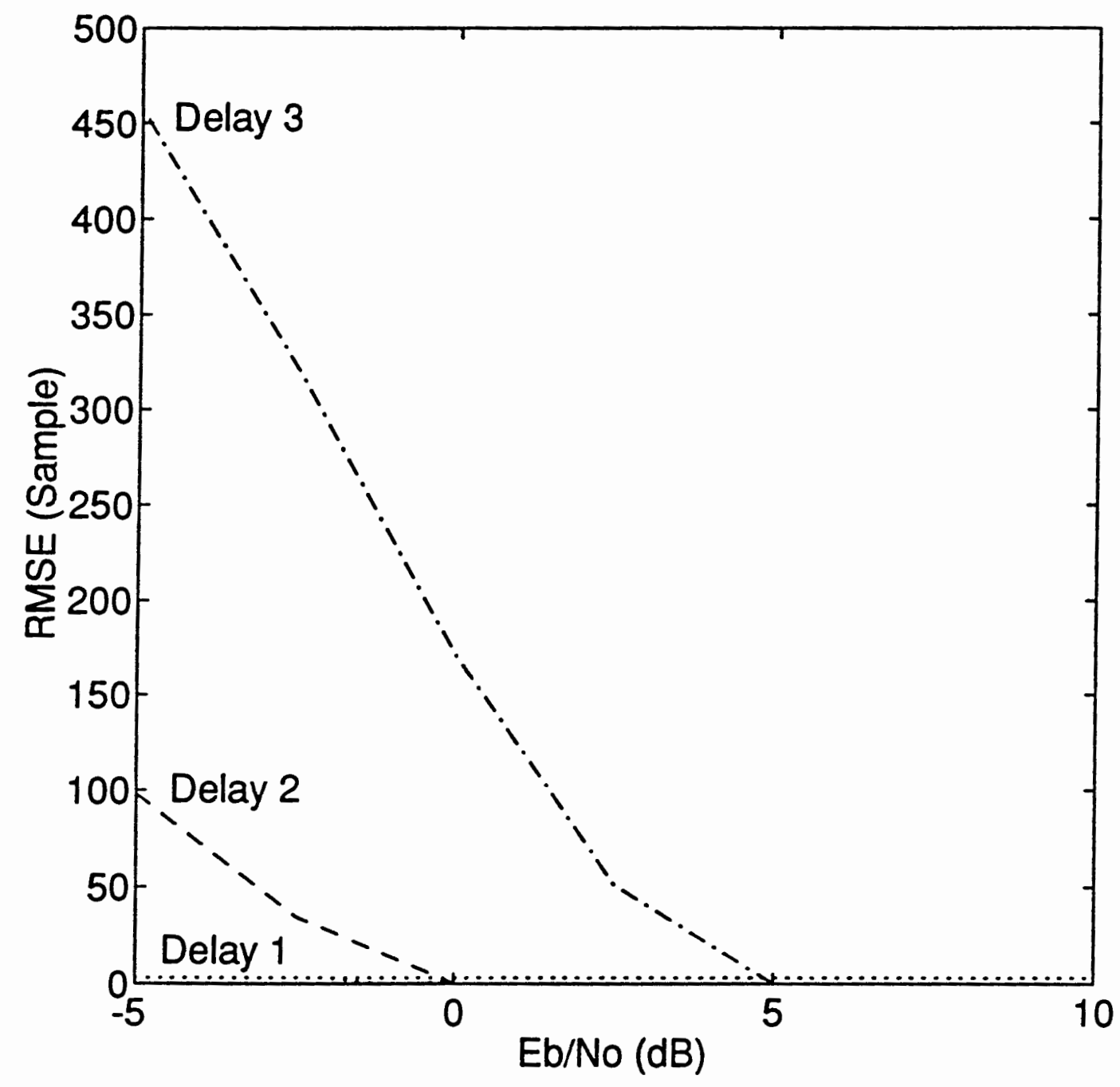

Fig. 6.4.2. Delay Estimation RMSE verse $E_{b} / N_{0}$ 


\section{VI.5 CONCLUSION}

In this chapter, we explore the possibility of using the advanced signal processing algorithms (using MUSIC and Min-Norm methods) to estimate the time delay of each path $\{\tau\}$. Clearly, those methods have better performance to estimate the time delay of each path in a realistic mobile environment. 


\section{CHAPTER VII}

\section{TAP WEIGHTS ESTIMATION}

\section{VII.1 INTRODUCTION}

In chapter V and chapter VI, we detect the number of delay paths (or taps) and estimate the time delay of each path. In the derivation of RAKE receiver [12], the channel tap weights are assumed known at receiver. However, to practically implement $R A K E$ receiver, the tap weights $\left\{c_{n}(t)\right\}$ need to be estimated.

To estimate the tap weights, earlier work [12] employed a single correlator. Its output is fed to the input of the low-pass filter after information-bearing signal is removed. To accomplish this, a delay of one signaling interval is introduced into the channel estimation procedure, as illustrated in Fig. 7.1.1. That is, first the receiver must decide whether the information in the received signal is +1 or -1 and, then, it uses the decision to remove the information from the correlator output prior to feeding it to the low-pass filter.

The objective of the chapter is to explore the possibility of using the advanced signal processing algorithms to estimate the multipath channels and to investigate the performance of the $R A K E$ receiver based on chip rate channel estimates in a realistic mobile environment.

Contributions included in this chapter are $[39,46]$ : 
1) A general description of diversity receiver

2) A new tap weight estimation approach for the multipath fading channel modeling using the signal processing algorithms.

3) The performance of the $R A K E$ receiver in a realistic mobile multipath fading channel $[25,26]$.

4) Simulation results on bit-error-rate (BER) as a function of $E_{b} / N_{0}$ using BPSK modulation for the multipath fading channel.

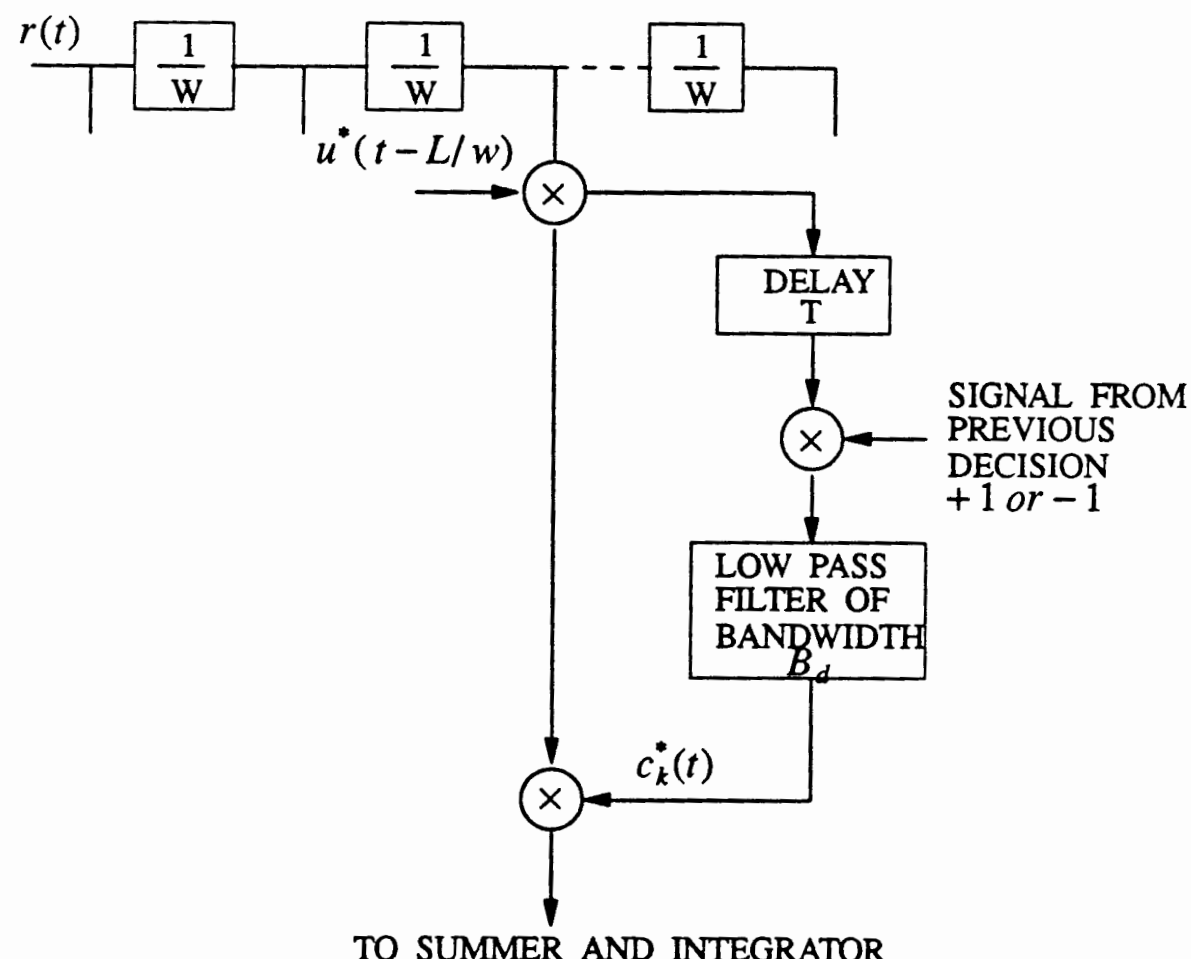

Fig. 7.1.1. Tap Weight Estimation 


\section{VII.2 DIVERSITY RECEIVER}

Based on the previous channel estimated for a tapped delay model, a diversity receiver (as in Fig. 7.2.1) [12] can be used to detect the signal from the multipath fading channel.

In a realistic mobile environment, the wide-band spread spectrum technique can be used to provide effective diversity gain to improve system performance.

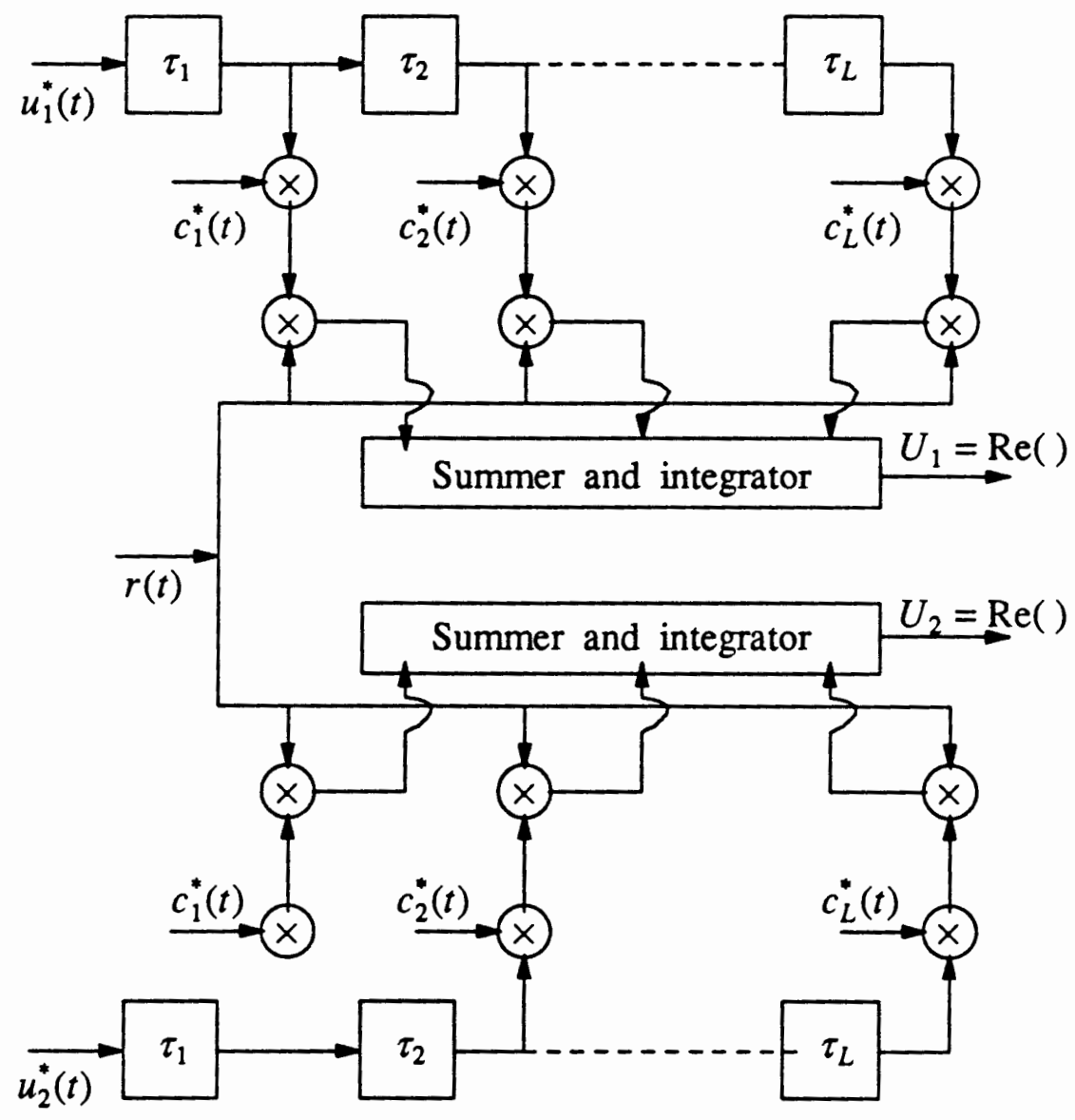

Fig. 7.2.1. Diversity Receiver 


\section{VII.3 NEW APPROACH}

We now propose the new tap weight estimation method for the RAKE receiver based on chip rate channel estimates in a realistic mobile environment. The wide-band spread spectrum technique can be used to provides effective diversity gain to improve system performance. In such a spread spectrum mobile communication system, when user information is transmitted through the traffic channel, there is always a companion pilot channel used as reference signal for channel estimation.

The binary phase shifted signal (BPSK) $m(t)$ with a given fundamental frequency $\omega_{0}$, is added, modulo -2 , by a binary sequence $b(t)$ from a pseudonoise (PN) code generator with much higher chip rate frequency. The sum $u(t)$ can then be used to modulate a carrier (Fig. 7.3.1).

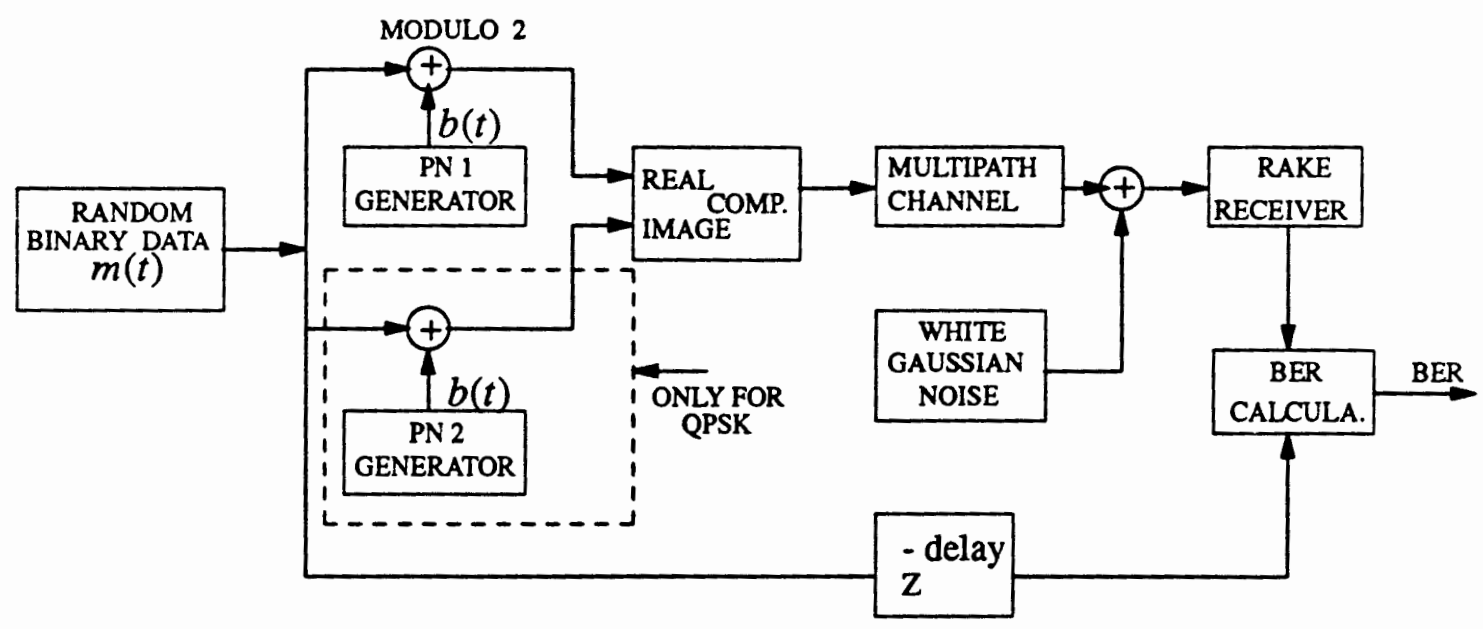

Fig. 7.3.1. Simulation Block Diagram 
For simplicity, we let the reference signal $v(t)$ in pilot channel to be constant 1 or -1 throughout, which also added, modulo - 2 , by the same PN sequence.

Without loss of generality, only the base-band modulation or demodulation is considered in this thesis.

Known symbols are inserted periodically into the data sequence prior to pulse shaping, and the composite signal is transmitted in the usual way over a channel characterized by multipath fading and additive noise.

Upon receiving the signals at receiver end, the pilot channel signal could be separated from the traffic channel signal through some well-known techniques, such as matched filter detection [38]. the pilot channel signal is used to estimate the tap weights.

The estimated tap weights are used for $R A K E$ receiver to recover the multipath fading signal in the traffic channel (Fig. 7.3.2).

There are two immediate consequences of the technique. The first is delay in the receiver; in order to obtain enough pilot samples for a good channel estimate, the receiver must wait and buffer samples for several symbols. The second is that the interpolation coefficients used to form the channel estimate depend on the position within the frame of the sample whose identity is to be determined.

The receiver must discard several data symbols at the start of each transmission, until it has accumulated enough pilot symbols for useful tap weights estimation. In here, we assume we have obtained enough pilot samples for a good channel estimation.

Using Eq.(4.2.9) (in chapter 4), the received pilot channel signal after multipath fading is

$$
s(t)=\sum_{n=1}^{L} c_{n}(t) v\left(t-\frac{n}{W}\right)+z(t)
$$


where for each symbol $t=1, \ldots, N$. Notice that $c_{n}$ is a constant within a symbol period $c_{n}(1)=c_{n}(N)=c_{n}$.

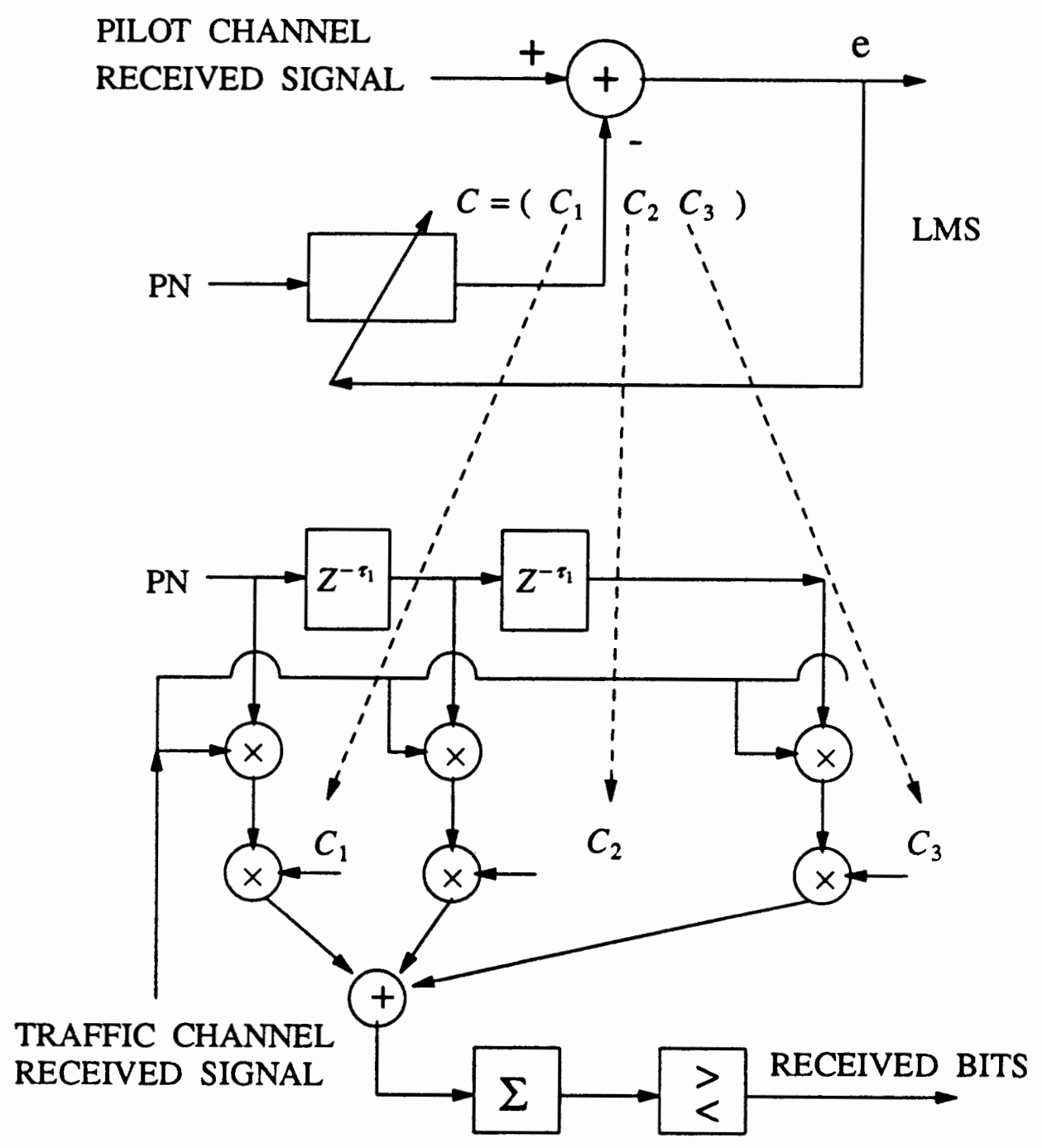

Fig. 7.3.2. Estimation Taps Weights using pilot channel 
In vector notation, Eq.(7.3.1) is

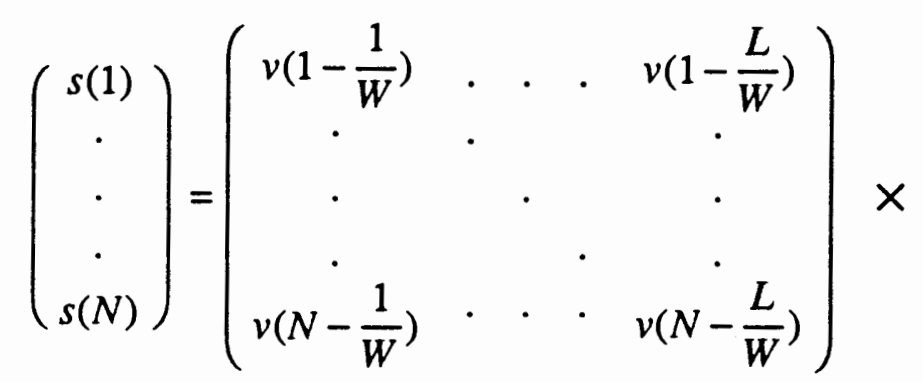

$$
\left(\begin{array}{c}
c_{1} \\
\cdot \\
\cdot \\
\cdot \\
c_{N}
\end{array}\right)+\left(\begin{array}{c}
z(1) \\
\cdot \\
\cdot \\
\cdot \\
z(N)
\end{array}\right)
$$

or equivalently, in vector notation

$$
s=V c+z
$$

To minimize $\|z\|^{2}=\|s-V c\|^{2}$ over $c$, We have

$$
\begin{aligned}
& \frac{\partial}{\partial c}(s-V c)^{H}(s-V c) \\
& V^{H}(s-V c)=0 \\
& V^{H} s=V^{H} V c
\end{aligned}
$$




$$
c=\left(V^{H} V\right)^{-1} V^{H} s=V^{\dagger} s
$$

We can now easily see that

$$
\hat{c}=V^{\dagger} s
$$

gives $\left\{C_{n}\right\}$ a least-square error (LSE) estimate at a given symbol time. In Eq.(7.3.8), the left pseudo-inverse $\dagger$ is defined as

$$
V^{\dagger}=\left(V^{H} V\right)^{-1} V^{H}
$$

$\hat{c}_{n}$ can now be used in RAKE receiver as in Fig. 7.2.1.

The estimation error covariance can be found as

$$
E_{z}\left[(\hat{c}-c)(\hat{c}-c)^{H}\right]=\sigma_{z}^{2}\left(V^{H} V\right)^{-1}
$$

\section{VII.4 PERFORMANCE COMPARISON}

In our computer simulations, we base on IS-95 standard and choose three paths with delays $\tau=1,7,20(T)$, respectively. where $T=\frac{1}{W}$. The ratio of amplitude attenuation of the second path over that of first path is $-3 \mathrm{~dB}$, and of third path over the first path is $-6 \mathrm{~dB}$.

Those equations are :

$$
20 \log \left[\frac{\left|C_{2}\right|}{\left|C_{1}\right|}\right]=-3 d B
$$




$$
20 \log \left[\frac{\left|C_{3}\right|}{\left|C_{1}\right|}\right]=-6 d B
$$

The fundamental frequency of the binary message signal $m(t)$ is chosen to be 19.2 $\mathrm{KHz}$, and the fundamental frequency of PN code sequence $b(t)$ is $64 \times 19.2 \mathrm{KHz}=1.2288 \mathrm{MHz}$.

We simulate the new approach in two different scenarios :

i) the pilot channel has the same energy-per-bit over noise-density $E_{b} / N_{0}$ as the traffic channel .

ii) the pilot channel has a constant $E_{b} / N_{0}$ of $20 \mathrm{~dB}$.

The Bit-Error-Rate (BER) verse $E_{b} / N_{0}$ of the $R A K E$ receiver is used to measure the performance, as shown in Fig. 7.4.1. In Fig. 7.4.1,

A) the BER verse $E_{b} / N_{0}$ of the RAKE receiver using the tap weight estimation shown in Fig. 7.4.1 in line A .

B) RAKE receiver using LSE tap weight estimates is plotted in line B for scenario (i).

C) RAKE receiver using LSE tap weight estimates is plotted in line C for scenario (ii ).

D) BER verse $E_{b} / N_{0}$ for receiver with exact channel tap weights is also shown in line D.

Clearly, LSE approach proposed in this thesis outperform the previous approach. 
At the same time, We can see that performance measured in BER of the RAKE receiver using LSE tap weights is very closed to that using exact weights.

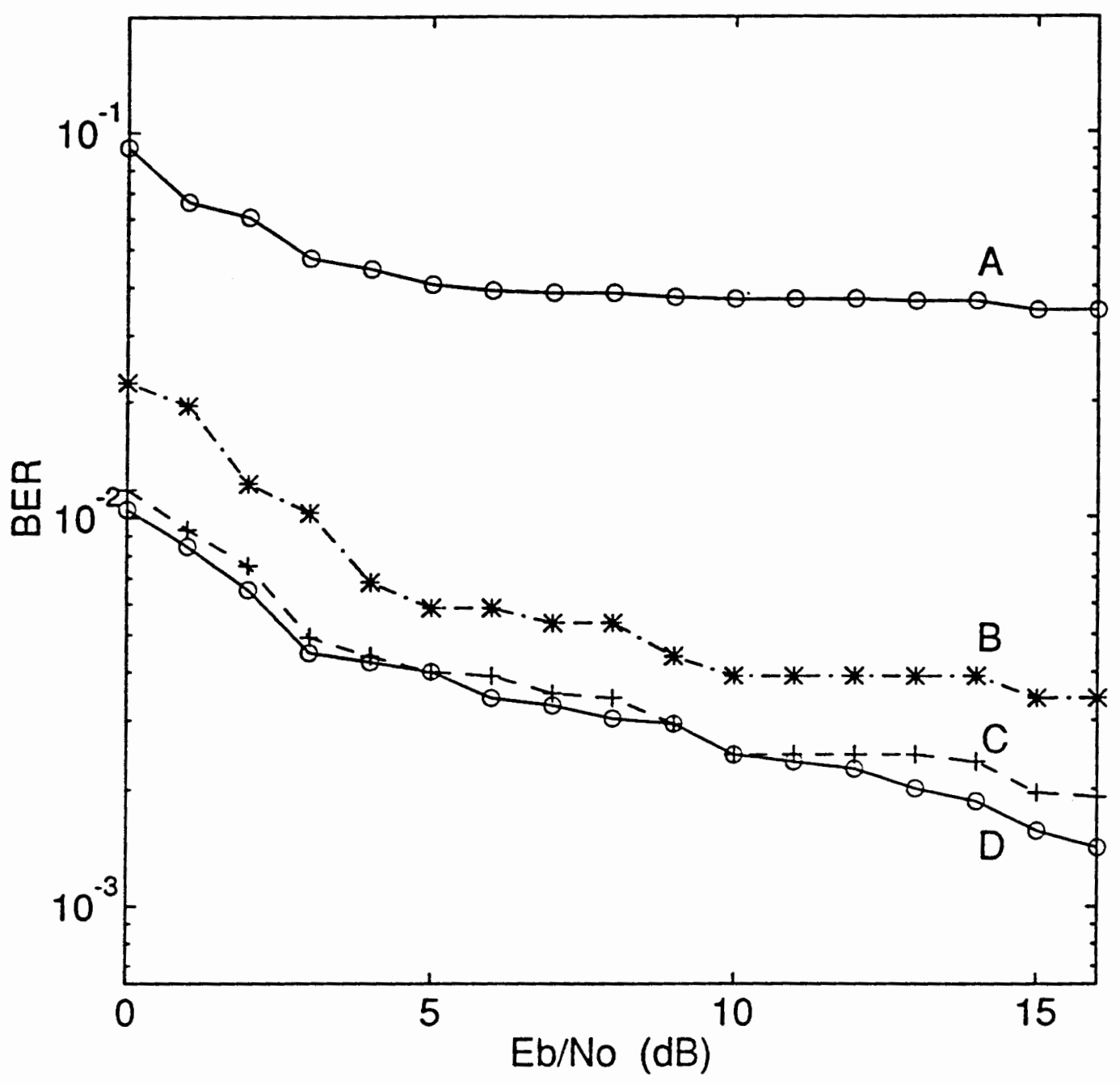

Fig. 7.4.1. Performance of BER verse $E_{b} / N_{0}$ 


\section{VII.5 CONCLUSION}

In this chapter, we explore the possibility of using the advanced signal processing algorithms to estimate the tap weights. Pilot channel is relatively simple to implement. Upon receiving the signals at receiver end, the pilot channel signal, which could be separated from the traffic channel signal through some well-known techniques, is used to estimate the tap weights. The estimated tap weights are used for $R A K E$ receiver to recover the multipath fading signal in the traffic channel. The performance measured in BER of the RAKE receiver using LSE tap weights is very closed to that using exact weights. Simulations show that the new approach outperforms the existing approaches. 


\section{CHAPTER VIII}

\section{CONCLUSION}

Multipath fading is one of the major practical concerns in wireless communications. Multipath problem always exists in mobile environment, especially for mobile unit which is often embedded in its surroundings. A time-variant tapped line delay model has been used for multipath fading in a wide-band spread spectrum mobile system.

In this thesis, we introduced mobile communications, fading signals, pilot channel, RAKE receiver, AIC and MDL methods, MUSIC and Min-Norm methods, and then we proposed to use the detection and estimation techniques developed in spectrum analysis and array processing to determine the number of delay paths (or taps), to estimate the time delay of each path, and to estimate tap weight of each delay path based on chip rate channel information in a realistic mobile environment.

Simulations show that the new approach outperforms the existing ansasches. 


\section{REFERENCES}

[1] Robert G. Winch, Telecommunication Transmission Systems: microwave, fiber optic, mobile cellular radio, data, and digital multiplexing, McGraw-Hill, Inc., 1993.

[2] William C. Y. Lee, Mobile Communications Design Fundamentals, John Wiley \& Sons, Inc., 1993.

[3] Davenport, W. B., and W. L. Root, Random Signals and Noise, McGraw-Hill, Inc., pp. $68,1958$.

[4] Lee, W. C. Y., and Y. S. Yeh, "On the Estimation of the Second-Order Statistics of Log-Normal Fading in Mobile Radio Environment," IEEE Trans. Commun. Com., Vol. 22, pp. 869-873, June 1974

[5] Kennedy, R. S., Fading Dispersive Communication Channels, Wiley-Interscience, ch.3, 1969.

[6] Rice, S. O., "Properties of Sine Wave Plus Random Noise," Bell Sys. Tech. J., Vol. 27, pp. 109-157, Jan. 1948.

[7] Lee, W. C. Y., "Statistical Analysis of the Level Crossings and Duration of Fades of the Signal from an Energy Density Mobile Radio Antenna," Bell Sys. Tech. J., Vol. 46, pp. 417-448, Feb. 1967.

[8] Cox, D. O., "Delay-Doppler Characteristics of Multipath Propagation at $910 \mathrm{MHz}$ in a Suburban Mobile Radio Environment," IEEE Trans. Antenna Propagation, Vol. 20, pp. 625-635, Sept. 1972. 
[9] Cox, D. O., and R. P. Leck, "Distribution of Multipath Delay Spread and Average Excess Delay for $910 \mathrm{MHz}$ Urban Mobile Radio Path," IEEE Trans. Antenna Propagation, Vol. 23, pp. 206-213, Mar. 1975.

[10]R. Price, and P. E. Green, Jr., "A Communication Technique for Multipath Channels," Proc. IRE, Vol. 46, pp. 555-570, Mar. 1958.

[11] Lindsay, D. G., "A Dual-Diversity Frequency-Shift Receiver," Proc. of the IRE, Vol. 22, pp. 598-612, June, 1951.

[12] J. G. Proakis, Digital Communications, New York, NY: McGraw-Hill, Inc., second ed., 1989.

[13]W. C. Jakes, Microwave Mobile Communications, New York, NY: John Wiley \& Sons, Inc., 1974.

[14] Gilhousen, K. S., I. M. Jacobs, R. Padovani, A. J. Viterbi, L. A. Weaver, and C. E. Wheatley, "On the Capacity of a Cellular CDMA System," IEEE Trans. Veh. Tech., Vol. 40, pp. 303-312, May 1991.

[15] Pickholtz, R. L., L. B. Milstein, and D. L. Schilling, "Spread Spectrum for Mobile Communications, " IEEE Trans. Veh. Tech., Vol. 40, pp. 313-322, May 1991.

[16] Viterbi, A. J., "When Not to Spread Spectrum - A Sequel, " IEEE Commun.23, pp. 12-17, Apr. 1985.

[17] Milstein, L. B., R. L. Pickholtz, and D. L. Schilling, "Optimization of the Processing Gain of an FSK - FH System, " IEEE Trans. Commun. COM - 28, pp. 1062-1079, July 1980 .

[18]Huth, G. K., "Optimization of Coded Spread Spectrum System Performance, " IEEE Trans. Commun. COM - 25, pp. 763-770, Aug. 1977.

[19] Simon, M. K., J. K. Omira, R. A. Scholtz, and B. K. Levin, Spread Spectrum Communications, Vol. 2, Rockville, MD: Computer Science Press, 1985. 
[20] Pickholtz, R. L., D. L. Schilling, L. B. Milstein, "Theory of Spread - Spectrum Communications - A Tutorial, " IEEE Trans. Commun. COM - 30, pp. 855-884, May 1982.

[21] Scholtz, R. A., "The origins of Spread Spectrum Communications, " IEEE Trans. Commun. COM - 30, pp. 882-884, May 1982.

[22] Viterbi, A. J., "Spread Spectrum Communications - Myths and Realities, "IEEE Commun., pp. 11-18, May 1979.

[23]Lee, W. C. Y., "Radio Access Technology - CDMA/Spread Spectrum, " seminar notes used for the one-day seminar of IEEE San Francisco Section/Pacific Bell at San Ramon, Ca. on Jan 23, 1990, and the seminar of IEEE New Jersey Section/ Rutgers Univ. WINLAB at Piscataway, N.J. on April 25, 1990.

[24]Lee, W. C. Y., "Overview of Cellular CDMA, " IEEE Trans. Veh. Tech., Vol. 40, pp. 291-302, May 1991.

[25] C. N. Pateros and G. J. Saulnier, "Adaptive Correlator Receiver Performance in Fading Multipath Channels, " Proc. 43rd IEEE Vehiclar Technology Conference, (New Jersey), pp. 746-749, May 1993.

[26] H. Erben and H. Nuszkowski, "Performance of RAKE Receiver in Realistic Mobile Radio Environment, " Proc. 43rd IEEE Vehiclar Technology Conference, (New Jersey), pp. 730-736, May 1993.

[27] G. L. Turin, "Introduction to Spread - Spectrum Antimultipath Techniques and their Applications to Urban Digital Radio, " Proc. IEEE, Vol. 68, pp. 328-353, March 1980.

[28]E. A. Geraniotis and M. B. Pursley, "Performance of Coherent Direct-Sequence Spread - Spectrum Communications over Specular Multipath Fading Channels, " IEEE Trans. Commun., Vol. 33, pp. 502-508, June 1985. 
[29] U. Grob, A. Welti, E. Zollinger, R. Kung, and H. Kaufmann, "Microcellular DirectSequence Spread Spectrum Radio System using n-path RAKE Receiver, " IEEE Journal on Selected Areas in Communications, Vol. 8, pp. 772-779, June 1990.

[30] J. P. McGeehan and A. J. Bateman, "Phase Locked Transparent Tone-in-Band (TTIB): A New Spectrum Configuration Particularly Suit to the Transmission of Data over SSB Mobile Radio Networks, " IEEE Trans. Commun., Vol. COM-32, pp. 81-87, Jan. 1984.

[31] A. J. Bateman et al., "Speech and Data Communications over $942 \mathrm{MHz}$ TAB and TTIB Signal Regeneration, " IEEE Trans. Veh. Tech., Vol. VT-34, pp. 13-21, Feb. 1985.

[32] F. Davarian, "Mobile Digital Communications via Tone Calibration, " IEEE Trans. Veh. Tech., Vol. VT-36, pp. 55-62, May 1987.

[33] P. M. Martin et al., "The Implementation of a 16-QAM Mobile Data System using TTIB-base Fading Correction Techniques, " in Proc. IEEE Veh. Tech. Conf., Philadelphia, PA, pp. 71-76, 1988.

[34] J. H. Lodge and M. L. Moher, "Time Diversity for Mobile Satellite Channels using Trellis Coded Modulations, " IEEE Global Telecommun. Conf., Tokyo, 1987.

[35] M. L. Moher and J. H. Lodge, "TCMP - a Modulation and Coding Strategy for Rician Fading Channels, " IEEE J. Select. Areas Commun., Vol. 7, pp. 1347-1355, Dec. 1989.

[36] S. Sampei and T. Sunaga, "Rayleigh Fading Compensation Method for 16-QAM in Digital Land Mobile Radio Channels, " in Proc. IEEE Veh. Tech. Conf., San Francisco, CA, pp. 640-646, May 1989.

[37] J. K. Cavers, "The Performance of Phase Locked Transparent Tone-in-Band with 
Symmetric Phase Detection, " IEEE Trans. Commun., Vol. 39, pp. 1389-1399, Sept. 1991.

[38] J. K. Cavers, "An Analysis of Pilot Symbol Assisted Modulation for Rayleigh Fading Channels, " IEEE Trans. Veh. Tech., Vol. 40, pp. 686-693, Nov. 1991.

[39] F. Li, H. Xiao, and J. Yang, "On Channel Estimation for RAKE Receiver in Mobile Multipath Fading Channel, " Proc. ICASSP'94, (Adelaide, Australia), April 1994.

[40] M. Kaveh, and H. Wang, Advances in Spectrum Analysis and Array Processing, ch. Threshold Properties of Narrow-Band Signal-Subspace Array Processing Methods, pp. 173-220, Eaglewood Cliffs, NJ: S. Haykin Ed., Prentice-Hall, 1985.

[41] H. Akaike, "A New Look at Statistical Model Identification, " IEEE Trans. Auto. Control, Vol. AC-19, NO.6, pp. 716-723, Dec. 1974.

[42] J. Rissanen, "Modeling by Shortest Data Description, " Automatica, Vol. 14, pp. 465-471, 1978.

[43] M. Wax and T. Kailath, "Detection of Signals by Information Theoretic Criteria, " IEEE Trans. Acoustics, Speech, and Signal Processing, Vol. ASSP-33, NO.2, pp. 387-392, April 1985.

[44] R. O. Schmidt, "Multiple Emitter Location and Signal Parameter estimation, " in Proc. RADC Spectral Estimation Workshop, (Griffiss AFG, NY), pp. 243-258, 1979.

[45] R. Kumaresan and D. W. Tufts, "Estimating the Angles of Arrival of Multiple Plane Waves, "IEEE Trans. on Aerospace and Electronic Systems, Vol. AES-19, pp. 134-139, Jan. 1983.

[46] F. Li, H. Xiao, Y. B. Guo, and J. Yang, "On Modeling of A Mobile Multipath Fading Channel, " Invited paper in IEEE 7th SP Workshop on Statistical Signal \& Array Processing, (Quebec City, Quebec, Canada), pp. 445-448, June 1994. 